\title{
Precision axion physics with running axion couplings
}

\author{
Kiwoon Choi, Sang Hui Im, Hee Jung Kim and Hyeonseok Seong \\ Center for Theoretical Physics of the Universe, Institute for Basic Science, \\ Daejeon 34126, South Korea \\ E-mail: kchoi@ibs.re.kr, imsanghui@ibs.re.kr, heejungkim@ibs.re.kr, \\ hseong@ibs.re.kr
}

ABSTRACT: We study the renormalization group running of axion couplings while taking into account that the Standard Model can be extended to its supersymmetric extension at a certain energy scale below the axion decay constant. We then apply our results to three different classes of axion models, i.e. KSVZ-like, DFSZ-like, and string-theoretic axions, and examine if string-theoretic axions can be distinguished from others by having a different pattern of low energy couplings to the photon, nucleons and electron. We find that the low energy couplings of string-theoretic axions have a similar pattern as those of KSVZ-like axions but yet reveal a sizable difference which might be testable in future axion search experiments. We also note that the coupling of KSVZ-like QCD axions to the electron is dominated by a three-loop contribution involving the exotic heavy quark, gluons, top quark and Higgs field.

Keywords: Beyond Standard Model, Effective Field Theories, Renormalization Group, Compactification and String Models

ARXiv EPrint: 2106.05816 


\section{Contents}

1 Introduction 1

2 Axion couplings to matter and gauge fields at UV scales 3

2.1 Field-theoretic axions: KSVZ-like and DFSZ-like axions 5

$\begin{array}{lll}2.2 & \text { String-theoretic axions } & 7\end{array}$

3 Running of the axion couplings $\quad 14$

$\begin{array}{lll}3.1 & \text { RG equations } & 14\end{array}$

$\begin{array}{lll}3.2 & \text { Semi-analytic solution and numerical results } & 21\end{array}$

4 Distinguishing the axions by low energy precision physics 24

$\begin{array}{llr}5 & \text { Conclusions } & 29\end{array}$

\section{Introduction}

Axions are one of the most compelling candidates for physics beyond the Standard Model (SM) of particle physics [1-3]. Axions were originally postulated to solve the strong CP problem [4-6] and later turned out to be a natural candidate for dark matter [7-9]. Generically there can also be axions not necessarily associated with the strong $\mathrm{CP}$ problem, which are often dubbed axion-like particle (ALP). Such ALPs can be much lighter than the QCD axion solving the strong $\mathrm{CP}$ problem and may constitute dark matter with interesting astrophysical signatures [10].

A key feature of axions is that the field variable is periodic as

$$
a(x) \cong a(x)+2 \pi f_{a},
$$

where the scale parameter $f_{a}$ is termed the axion decay constant. Axions can be naturally light as their couplings are constrained by an approximate non-linear $\mathrm{U}(1)_{\mathrm{PQ}}$ symmetry which involves a shift of the axion field:

$$
\mathrm{U}(1)_{\mathrm{PQ}}: \quad a(x) \rightarrow a(x)+\text { constant. }
$$

Axions may originate from the phase of complex scalar field $[4-6,11-14]$ as

$$
\sigma(x)=\rho(x) e^{i a(x) / f_{a}}
$$

and then $f_{a}$ is determined by the vacuum expectation value of $\rho$ as $f_{a}=\sqrt{2}\langle\rho\rangle$. In the following discussion, such axions will be termed "field-theoretic axions". It is obvious that the field region around $f_{a}=0$ for field-theoretic axions is well described by an effective field theory in which $\mathrm{U}(1)_{\mathrm{PQ}}$ is linearly realized. There are two types of field-theoretic axion models 
which are phenomenologically viable and have been widely discussed in the literature: ${ }^{1}$ the Kim-Shifman-Vainshtein-Zakharov (KSVZ) axion models [11, 12] in which all SM fields are neutral under the linearly-realized $\mathrm{U}(1)_{\mathrm{PQ}}$ and the Dine-Fischler-Srednicki-Zhitnitsky (DFSZ) axion models $[13,14]$ in which the SM fermions carry nonzero $\mathrm{U}(1)_{\mathrm{PQ}}$-charges.

Axions can also arise from the zero modes of a higher-dimensional $p$-form gauge field $C^{(p)}$ which has a quantized coupling to $(p-1)$-brane in the underlying UV theory $[16,17]$ :

$$
a(x)=\int_{\Sigma_{p}} C_{\left[m_{1}, \ldots, m_{p}\right]}^{(p)}(x, y) d y^{m_{1}} \ldots d y^{m_{p}},
$$

where $\Sigma_{p}$ is a $p$-dimensional cycle in the compact internal space with the coordinates $y^{m}$. We will term such axions originated from $C^{(p)}$ "string-theoretic axions". It is widely believed that the limit with $f_{a}=0$ for string-theoretic axions is in swampland at an infinite field distance with infinite towers of light particles [18].

It is well known that KSVZ axions and DFSZ axions have a different pattern of low energy couplings, which might be testable in future axion search experiments $[1,3]$. For instance, at tree level, the couplings of KSVZ axions to the SM fermions are all vanishing, while those of DFSZ axions are of order unity in the unit of $1 / f_{a}$, which results in different ratios among the low energy axion couplings $g_{a X}(X=\gamma, p, n, e)$ to the photon, nucleons and electron. Similarly, generic DFSZ-like axions with $\mathcal{O}(1)$ tree-level couplings (again in the unit of $\left.1 / f_{a}\right)$ to the light quarks and electron have a clearly distinguishable pattern of $g_{a X}(X=\gamma, p, n, e)$ compared to those of generic KSVZ-like axions with negligible tree-level couplings to the light quarks and electron. On the other hand, it has not been examined yet if string-theoretic axions can also be distinguished from other types of axions by their low energy couplings. As we will see, the couplings of string-theoretic axions to the SM gauge and matter fields have a similar pattern as those of KSVZ-like axions. Therefore it requires a careful analysis including radiative corrections [15, 19-25] to axion couplings to see if string-theoretic axions can be discriminated from KSVZ-like axions.

With this motivation, in this paper, we study the renormalization group (RG) running of axion couplings in a generic context and apply the results to the models of our interest. To examine if string-theoretic axions and KSVZ-like axions can have a distinguishable pattern of $g_{a X}(X=\gamma, p, n, e)$, we first perform a generic renormalization group analysis for the axion couplings to matter fermions and Higgs fields while taking into account that the Standard Model can be extended to its supersymmetric extension at a scale below $f_{a}$. We then find that string-theoretic QCD axion and KSVZ-like QCD axion have a significantly different value of the coupling ratio $g_{a e} / g_{a \gamma}$, which would make it feasible to distinguish one from the other by an experimental measurement of $g_{a e} / g_{a \gamma}$. For an axionlike particle (ALP) which does not couple to the gluons but has a non-zero coupling to the photon, the prospect for discrimination is much more promising: all of the three coupling ratios $g_{a Y} / g_{a \gamma}(Y=p, n, e)$ for string-theoretic ALP and KSVZ-like ALP differ by about one order of magnitude. We also find that the coupling of KSVZ-like QCD axion to the

\footnotetext{
${ }^{1}$ Here we consider only relatively simple models for field-theoretic axions. For a discussion of more involved models, e.g. those with hierarchical axion couplings, see for instance [15].
} 
electron is dominated by a three-loop contribution involving the heavy exotic quark, gluons, top quarks and Higgs doublet, which was not noticed in the previous studies [19-25].

The organization of this paper is as follows. In section 2 , we discuss the relevant features of the axion couplings to gauge and matter fields at the UV boundary scale $\mu \sim f_{a}$ for DFSZ-like, KSVZ-like and string-theoretic axions. In section 3, we present the RG equations for axion couplings in the context of generic effective lagrangian and compute the resulting radiative corrections to the low energy axion couplings to the light quarks and electron. In this analysis, we take into account that the SM can be extended to its supersymmetric extension at an energy scale below $f_{a}$. In section 4 , we apply our result to the three different classes of axions, i.e. DFSZ-like, KSVZ-like, and string-theoretic axions, to see if they can be distinguished from each other by their low energy couplings to the photon, nucleons and electron. Section 5 is the conclusion.

\section{Axion couplings to matter and gauge fields at UV scales}

In this section, we discuss the axion couplings to gauge and matter fields at the UV boundary scale $\mu \sim f_{a}$ in models of KSVZ-like, DFSZ-like, and string-theoretic axions. We will be brief on field-theoretic KSVZ-like and DFSZ-like axions and pay more attention to the characteristic features of string-theoretic axions. To proceed, we adopt the Georgi-KaplanRandall (GKR) field basis [26] which can be defined at a scale around or below $f_{a}$, for which only the axion field transforms under $\mathrm{U}(1)_{\mathrm{PQ}}$ as

$$
\mathrm{U}(1)_{\mathrm{PQ}}: \quad a(x) \rightarrow a(x)+\text { constant, }
$$

and all other fields in the model are invariant under $\mathrm{U}(1)_{\mathrm{PQ}}$. Then the relevant axion couplings at $\mu \sim f_{a}$ take the form

$$
\frac{1}{2} \partial_{\mu} a \partial^{\mu} a+\frac{\partial_{\mu} a}{f_{a}}\left[i c_{\phi}\left(\phi^{*} D^{\mu} \phi-\phi D^{\mu} \phi^{*}\right)+c_{\psi} \bar{\psi} \bar{\sigma}^{\mu} \psi\right]+c_{A} \frac{g_{A}^{2}}{32 \pi^{2}} \frac{a(x)}{f_{a}} F^{A \mu \nu} \tilde{F}_{\mu \nu}^{A},
$$

where $\phi$ and $\psi$ denote canonically normalized gauge-charged scalar fields and chiral fermions in the model, and $F_{\mu \nu}^{A}=\left(G_{\mu \nu}^{\alpha}, W_{\mu \nu}^{i}, B_{\mu \nu}\right)$ are the canonically normalized gauge field strength of the $\mathrm{SM}$ gauge group $\mathrm{SU}(3)_{c} \times \mathrm{SU}(2)_{L} \times \mathrm{U}(1)_{Y}$. In this description, the axion couplings $c_{A}(A=G, W, B)$ to gauge fields are quantized, e.g. integer-valued for an appropriate normalization of the gauge couplings $g_{A}^{2}$, and invariant under the subsequent RG evolution, while the couplings $c_{\phi}$ and $c_{\psi}$ to matter fields are real-valued and generically have nontrivial RG evolution induced by the gauge and Yukawa interactions in the model.

As will be discussed in detail in section 3, to examine the low energy axion couplings in the model, one can first scale the effective lagrangian (2.2) down to a low energy scale $\mu=\mathcal{O}(1) \mathrm{GeV}$. The relevant axion couplings at this scale are given by

$$
\frac{1}{32 \pi^{2}} \frac{a(x)}{f_{a}}\left(c_{\gamma} e^{2} F^{\mu \nu} \tilde{F}_{\mu \nu}+c_{G} g_{s}^{2} G^{\alpha \mu \nu} \tilde{G}_{\mu \nu}^{\alpha}\right)+\sum_{\Psi=u, d, e} \frac{\partial_{\mu} a}{2 f_{a}} C_{\Psi} \bar{\Psi} \gamma^{\mu} \gamma_{5} \Psi
$$

where $e$ and $g_{s}$ are the electromagnetic and color gauge couplings, respectively, and

$$
c_{\gamma}=c_{W}+c_{B}, \quad C_{\Psi}=C_{\Psi}^{0}+\Delta C_{\Psi},
$$


with

$$
\begin{aligned}
& C_{u}^{0}=c_{Q_{1}}\left(f_{a}\right)+c_{u_{1}^{c}}\left(f_{a}\right)+c_{H}\left(f_{a}\right), \\
& C_{d}^{0}=c_{Q_{1}}\left(f_{a}\right)+c_{d_{1}^{c}}\left(f_{a}\right)-c_{H}\left(f_{a}\right), \\
& C_{e}^{0}=c_{L_{1}}\left(f_{a}\right)+c_{e_{1}^{c}}\left(f_{a}\right)-c_{H}\left(f_{a}\right) .
\end{aligned}
$$

Here $C_{\Psi}^{0}(\Psi=u, d, e)$ denote the axion couplings to the light quarks and electron evaluated at tree-level, which can be interpreted as the couplings at the UV boundary scale $\mu=f_{a}$ in our approximation, $\Delta C_{\Psi}$ are radiative corrections to $C_{\Psi}$ received over the scales from $f_{a}$ to $\mu=\mathcal{O}(1) \mathrm{GeV}$, which will be extensively discussed in section $3, c_{\phi}\left(f_{a}\right)$ and $c_{\psi}\left(f_{a}\right)$ are the axion couplings at $\mu=f_{a}$ including the couplings to the Higgs doublet $H$ and the three generations of chiral quarks and leptons $\psi=\left\{Q_{i}, u_{i}^{c}, d_{i}^{c}, L_{i}, e_{i}^{c}\right\}(i=1,2,3)$, which would be determined by the underlying UV-completed axion model, and the effects of flavor mixing are ignored for simplicity. The axion-Higgs coupling $c_{H}$ gives rise to the axion couplings to the SM fermions via the axion- $Z$ boson kinetic mixing $\partial_{\mu} a Z^{\mu}$ below the weak scale. Alternatively, one can rotate away the axion-Higgs coupling $c_{H}$ to the axion-fermion couplings by performing axion-dependent field redefinition proportional to $\mathrm{U}(1)_{Y}$ hypercharge:

$$
H \rightarrow H e^{i c_{H} a / f_{a}}, \quad \psi \rightarrow \psi e^{i\left(Y_{\psi} / Y_{H}\right) c_{H} a / f_{a}},
$$

which results in eq. (2.5).

In regard to the experimental verification of axions, the most relevant couplings are those to the photon, nucleons and electron at scales well below $\mathrm{GeV}$ :

$$
\frac{1}{4} g_{a \gamma} a \vec{E} \cdot \vec{B}+\partial_{\mu} a\left[\frac{g_{a e}}{2 m_{e}} \bar{e} \gamma^{\mu} \gamma_{5} e+\frac{g_{a n}}{2 m_{n}} \bar{n} \gamma^{\mu} \gamma_{5} n+\frac{g_{a p}}{2 m_{p}} \bar{p} \gamma^{\mu} \gamma_{5} p\right]
$$

which are determined by the couplings in (2.3) as follows:

$$
\begin{aligned}
g_{a \gamma} & \simeq \frac{\alpha_{\mathrm{em}}}{2 \pi} \frac{1}{f_{a}}\left(c_{\gamma}-\frac{2}{3} \frac{m_{u}+4 m_{d}}{m_{u}+m_{d}} c_{G}\right) \simeq \frac{\alpha_{\mathrm{em}}}{2 \pi} \frac{1}{f_{a}}\left(c_{\gamma}-1.92 c_{G}\right), \\
g_{a p} & \simeq \frac{m_{p}}{f_{a}}\left(C_{u} \Delta u+C_{d} \Delta d-\left(\frac{m_{d}}{m_{u}+m_{d}} \Delta u+\frac{m_{u}}{m_{u}+m_{d}} \Delta d\right) c_{G}\right), \\
& \simeq \frac{m_{p}}{f_{a}}\left(0.90 C_{u}(2 \mathrm{GeV})-0.38 C_{d}(2 \mathrm{GeV})-0.48 c_{G}\right), \\
g_{a n} & \simeq \frac{m_{n}}{f_{a}}\left(C_{d} \Delta u+C_{u} \Delta d-\left(\frac{m_{u}}{m_{u}+m_{d}} \Delta u+\frac{m_{d}}{m_{u}+m_{d}} \Delta d\right) c_{G}\right), \\
& \simeq \frac{m_{n}}{f_{a}}\left(0.90 C_{d}(2 \mathrm{GeV})-0.38 C_{u}(2 \mathrm{GeV})-0.04 c_{G}\right), \\
g_{a e} & \simeq \frac{m_{e}}{f_{a}} C_{e}\left(m_{e}\right),
\end{aligned}
$$

with $\Delta u=0.897(27)$ and $\Delta d=-0.376(27)$ at $\mu=2 \mathrm{GeV}$ in $\overline{\mathrm{MS}}$, and $m_{u} / m_{d}=0.48(3)[3$, 27]. One of our primary concerns is if string-theoretic axions can be discriminated from field-theoretic axions by having a distinguishable pattern of $g_{a X}(X=\gamma, p, n, e)$. 


\subsection{Field-theoretic axions: KSVZ-like and DFSZ-like axions}

Field-theoretic axion models have a UV completion with a linearly realized Peccei-Quinn symmetry:

$$
\mathrm{U}(1)_{\mathrm{PQ}}: \quad \sigma \rightarrow e^{i \alpha} \sigma, \quad \phi \rightarrow e^{i q_{\phi} \alpha} \phi, \quad \psi \rightarrow e^{i q_{\psi} \alpha} \psi
$$

where $\sigma$ denotes a $\mathrm{U}(1)_{\mathrm{PQ}}$-charged complex scalar field whose phase field is identified as the axion as in (1.3). For simplicity, here we assume that $\mathrm{U}(1)_{\mathrm{PQ}}$ is spontaneously broken predominantly by the vacuum expectation value of a single gauge-singlet complex scalar field $\sigma$.

To move to the GKR field basis [26], one can parameterize $\sigma$ as

$$
\sigma=\frac{1}{\sqrt{2}}\left(f_{a}+\hat{\rho}\right) e^{i a / f_{a}},
$$

where $\langle\hat{\rho}\rangle=0$, and make an axion-dependent field redefinition:

$$
\phi \rightarrow e^{-i q_{\phi} a / f_{a}} \phi, \quad \psi \rightarrow e^{-i q_{\psi} a / f_{a}} \psi
$$

Then, $\mathrm{U}(1)_{\mathrm{PQ}}$ is realized as the GKR form in (2.1), and the axion couplings to gauge and matter fields arise as a consequence of the field redefinition (2.11). The resulting couplings at $\mu \sim f_{a}$ are determined simply by the $\mathrm{PQ}$-charges of the linear $\mathrm{U}(1)_{\mathrm{PQ}}$ symmetry (2.9):

$$
c_{\phi}\left(f_{a}\right)=q_{\phi}, \quad c_{\psi}\left(f_{a}\right)=q_{\psi}, \quad c_{A}=\sum_{\psi} 2 q_{\psi} \operatorname{Tr}\left(T_{A}^{2}(\psi)\right),
$$

where $T_{A}(\psi)(A=G, W, B)$ denotes the gauge charge of $\psi$ for the SM gauge group $\mathrm{SU}(3)_{c} \times$ $\mathrm{SU}(2)_{L} \times \mathrm{U}(1)_{Y}$.

There are two different types of field-theoretic axion models that have been widely discussed in the literature, DFSZ models in which the SM fermions carry non-zero U(1) ${ }_{\mathrm{PQ}}$ charges and KSVZ models in which all SM fermions are neutral under $\mathrm{U}(1)_{\mathrm{PQ}}$. A minimal non-supersymmetric (non-SUSY) DFSZ model $[13,14]$ involves the two Higgs doublets $H_{u}$ and $H_{d}$ which have the Yukawa couplings for the up-type quark masses and the down-type quark and lepton masses, respectively, as well as the coupling $\kappa \sigma^{2} H_{u} H_{d}$ in the potential with $\kappa$ small enough to allow the correct electroweak symmetry breaking. The resulting axion couplings at $\mu \sim f_{a}$ are given by

$$
\text { non-SUSY DFSZ : } \quad c_{H_{u, d}}\left(f_{a}\right)=-1, \quad c_{\psi}\left(f_{a}\right)=\frac{1}{2}, \quad c_{G}=c_{W}=\frac{3}{5} c_{B}=6,
$$

where $\psi=\left\{Q_{i}, u_{i}^{c}, d_{i}^{c}, L_{i}, e_{i}^{c}\right\}$ are the chiral quarks and leptons in the SM. The corresponding tree-level axion couplings to the light quarks and electron, which can be identified as the couplings at $\mu \sim f_{a}$, are

$$
\text { DFSZ : } \quad C_{u}^{0}=2 \cos ^{2} \beta, \quad C_{d}^{0}=C_{e}^{0}=2 \sin ^{2} \beta,
$$

where $\tan \beta=\left\langle H_{u}\right\rangle /\left\langle H_{d}\right\rangle$ and we used $c_{H}=c_{H_{u}} \sin ^{2} \beta-c_{H_{d}} \cos ^{2} \beta$ for the coupling to the SM Higgs doublet $H$. It is straightforward to supersymmetrize this model, for instance 
with a superpotential involving a term $\sim \sigma^{2} H_{u} H_{d} / M_{P}[28]$, where $M_{P}=2.4 \times 10^{18} \mathrm{GeV}$ is the reduced Planck scale. The axion couplings of this SUSY DFSZ model at $\mu \sim f_{a}$ are

SUSY DFSZ : $\quad c_{H_{u, d}}\left(f_{a}\right)=-1, \quad c_{\psi}\left(f_{a}\right)=\frac{1}{2}, \quad c_{G}=6, \quad c_{W}=4, \quad c_{B}=8$,

where now $H_{u, d}$ and $\psi$ denote the full supermultiplets including superpartners, and $c_{W, B}$ includes the Higgsino contribution to the $\mathrm{U}(1)_{\mathrm{PQ}}$ anomaly. Obviously, the tree-level axion couplings to the light quarks and electron in this SUSY DFSZ model are the same as those of its non-SUSY counterpart which is given by (2.14).

In KSVZ models $[11,12]$, all SM fields are neutral under $\mathrm{U}(1)_{\mathrm{PQ}}$. However, there exist exotic $\mathrm{U}(1)_{\mathrm{PQ}}$-charged left-handed quark and anti-quark $\left(\mathcal{Q}, \mathcal{Q}^{c}\right)$ which get a heavy mass as a consequence of the spontaneous breakdown of $\mathrm{U}(1)_{\mathrm{PQ}}$ by the vacuum expectation value $\langle\sigma\rangle=f_{a} / \sqrt{2}$, e.g. get a mass by the Yukawa coupling

$$
\mathcal{L}_{\text {Yukawa }}=y_{\mathcal{Q}} \sigma \mathcal{Q} \mathcal{Q}^{c}
$$

One then finds

$$
\mathrm{KSVZ}: \quad c_{\mathcal{Q}}\left(f_{a}\right)=c_{\mathcal{Q}^{c}}\left(f_{a}\right)=\frac{1}{2}, \quad c_{H}\left(f_{a}\right)=c_{\psi}\left(f_{a}\right)=0, \quad c_{A}=2 \operatorname{Tr}\left(T_{A}^{2}(\mathcal{Q})\right)
$$

for $\psi=\left\{Q_{i}, u_{i}^{c}, d_{i}^{c}, L_{i}, e_{i}^{c}\right\}$, which result in

$$
\text { KSVZ : } \quad C_{\Psi}^{0}=0 \quad(\Psi=u, d, e) .
$$

In fact, there can be a contribution to $c_{\psi}\left(f_{a}\right)$ from Planck-scale-suppressed higher-dimensional operator such as $\sigma^{*} \partial_{\mu} \sigma \bar{\psi} \sigma^{\mu} \psi / M_{P}^{2}$, which would give $c_{\psi}\left(f_{a}\right)=\mathcal{O}\left(f_{a}^{2} / M_{P}^{2}\right)$, and therefore

$$
C_{\Psi}^{0}=\mathcal{O}\left(\frac{f_{a}^{2}}{M_{P}^{2}}\right) \lesssim \mathcal{O}\left(\left(\frac{g_{\mathrm{GUT}}^{2}}{8 \pi^{2}}\right)^{2}\right),
$$

where $g_{\text {GUT }}$ denotes the SM gauge coupling around the GUT scale or $f_{a}$, and we use the axion weak gravity conjecture bound $f_{a} / M_{P} \lesssim \mathcal{O}\left(g_{\text {GUT }}^{2} / 8 \pi^{2}\right)$ [29] for the last inequality. As in the case of DFSZ model, it is straightforward to supersymmetrize the above KSVZ model and the resulting SUSY model also has vanishing tree-level couplings to the SM Higgs and fermion fields.

One can consider more generic models of field-theoretic axions other than those presented above, e.g. models with different Higgs sector for the Yukawa couplings of the $\mathrm{SM}$ fermions, or additional $\mathrm{U}(1)_{\mathrm{PQ}}$-charged fields, or even flavor-dependent $\mathrm{U}(1)_{\mathrm{PQ}}$-charges [30-37]. In the following, we use the term "DFSZ-like axions" for generic field-theoretic axions with $C_{\Psi}^{0}=\mathcal{O}(1)(\Psi=u, d, e)$ and the term "KSVZ-like axions" for those with $C_{\Psi}^{0}=0$ (or more generically $C_{\Psi}^{0} \lesssim \mathcal{O}\left(\left(g_{\mathrm{GUT}}^{2} / 8 \pi^{2}\right)^{2}\right)$. As is well known (e.g. see table 1 in [38]), DFSZ-like and KSVZ-like axions have a clearly different pattern of the observable low energy couplings $g_{a X}(X=\gamma, p, n, e)$, so can be easily distinguished from each other. 


\subsection{String-theoretic axions}

String theory involves a variety of extended objects ((p-1)-branes) which have quantized couplings to a $p$-form gauge field in the theory [39]. As a consequence, string models accommodating the SM generically contain a string-theoretic axion that couples to the SM gauge and matter fields in 4-dimensional effective theory [16, 17]. Such axions are accompanied by their scalar partners, i.e. saxions or moduli $\tau$, whose vacuum expectation value can be identified as the Euclidean action of the brane instanton which has a quantized coupling to axion.

Generically there can be multiple string-theoretic axions, sometimes many [17]. Yet the low energy consequence of those axions depends crucially on the axion masses which have a close connection with the mechanism to stabilize their moduli partners. For instance, many of string-theoretic axions may get a heavy mass together with their moduli partners and therefore are decoupled from the low energy world. On the other hand, in this paper we are mostly interested in light axions whose couplings to the SM can be probed by precision low energy experiments, which include a QCD axion and an ultralight ALP coupled to the photon. Such light axions are required to be much lighter than their moduli partners, so we need a mechanism to stabilize moduli while keeping some axions to be nearly massless. In the following, we simply assume that underlying string model provides such a mechanism as was discussed for instance in [40-45].

Usually, string-theoretic axion and its modulus partner $\tau$ form a complex scalar field which corresponds to the scalar component of a chiral superfield in 4-dimensional effective supergravity of the underlying string compactification:

$$
T=\tau(x)+i \frac{a(x)}{f_{a}} .
$$

Then the axion couplings to gauge and matter fields are determined by the moduli Kahler potential $K_{0}$, the matter Kahler metric $Z_{I}$, and the holomorphic gauge kinetic functions $\mathcal{F}_{A}$ of the model, which are given by

$$
\begin{aligned}
K & =K_{0}\left(T+T^{*}\right)+Z_{I}\left(T+T^{*}\right) \Phi_{I}^{*} \Phi_{I}+\ldots, \\
\mathcal{F}_{A} & =\frac{c_{A}}{8 \pi^{2}} T+\ldots,
\end{aligned}
$$

where $\Phi_{I}$ denote gauge-charged chiral matter superfields in the model, and the ellipses stand for the terms higher order in $\Phi_{I}$, as well as the non-perturbative $\mathrm{U}(1)_{\mathrm{PQ}}$-breaking terms which would be exponentially suppressed by $e^{-T}$. Here, for simplicity, we assume a diagonal form of the matter Kahler metric $Z_{I}$ and ignore moduli superfields other than $T$. Note that the factor $1 / 8 \pi^{2}$ in $\mathcal{F}_{\mathcal{A}}$ appears due to the normalization convention of $T$ for which $\operatorname{Im}(T)=a(x) / f_{a} \cong \operatorname{Im}(T)+2 \pi$, and $c_{A}$ are rational numbers (or integers) in this convention. It is then straightforward to find that the decay constant and couplings of the axion $a(x)$ are given by

$$
\frac{1}{2} f_{a}^{2}=M_{P}^{2} \frac{\partial^{2} K_{0}}{\partial T \partial T^{*}}
$$

and

$$
c_{\phi_{I}}=\frac{\partial \ln Z_{I}}{\partial T}, \quad c_{\psi_{I}}=\frac{\partial \ln \left(e^{-K_{0} / 2} Z_{I}\right)}{\partial T}, \quad c_{A}=8 \pi^{2} \frac{\partial}{\partial T} \mathcal{F}_{A},
$$


where $\phi_{I}$ and $\psi_{I}$ denote the scalar component and the fermion component, respectively, of the chiral superfield $\Phi_{I}$.

The vacuum value of $\tau$ can be constrained in several ways. As noticed above, $\tau$ corresponds to the Euclidean action of a brane instanton which couples to $a(x)$, and then the axion weak gravity conjecture (WGC) [29] implies

$$
\tau \lesssim \mathcal{O}\left(\frac{M_{P}}{f_{a}}\right)
$$

Generically this brane instanton contributes to the axion potential, which can be parameterized as

$$
\delta V=e^{-\tau} \Lambda_{\mathrm{ins}}^{2} M_{P}^{2} \cos \left(\frac{a}{f_{a}}\right),
$$

where $\Lambda_{\text {ins }}$ is a model-dependent mass parameter determined by a more detailed feature of the model, in particular by the fermion zero modes of the brane instanton and the scale of supersymmetry breaking. In fact, explicit examples in string theory suggest that those brane instantons induce a correction of $\mathcal{O}\left(e^{-T}\right)$ to the superpotential or to the Kahler potential of the 4-dimensional effective supergravity [46, 47], which results in ${ }^{2}$

$$
\Lambda_{\text {ins }}^{2}=\mathcal{O}\left(m_{3 / 2} M_{P}\right) \text { or } \mathcal{O}\left(m_{3 / 2}^{2}\right) .
$$

At any rate, the brane-instanton-induced potential (2.25) provides a lower bound on the axion mass:

$$
m_{a}^{2} \gtrsim e^{-\tau} \Lambda_{\mathrm{ins}}^{2} M_{P}^{2} / f_{a}^{2}
$$

which implies that the brane instanton action is bounded from below as

$$
\tau \gtrsim 2 \ln \left(\Lambda_{\text {ins }} / m_{a}\right)
$$

For a string-theoretic QCD axion, the brane instanton-induced axion potential (2.25) should be further suppressed not to spoil the axion solution to the strong CP problem. Specifically one needs

$$
\delta V \lesssim 10^{-10} m_{\pi}^{2} f_{\pi}^{2}
$$

implying

$$
\tau \gtrsim \ln \left(10^{10} \Lambda_{\mathrm{ins}}^{2} / f_{\pi} m_{\pi}\right)
$$

for a QCD axion, where $m_{\pi}$ and $f_{\pi}$ are the pion mass and the pion decay constant, respectively.

On the other hand, for $c_{A}$ of order unity, $\tau$ is also bounded from above:

$$
\tau \lesssim \mathcal{O}\left(\frac{8 \pi^{2}}{g_{\mathrm{GUT}}^{2}}\right)
$$

\footnotetext{
${ }^{2}$ For models with a string scale $M_{\text {st }} \ll M_{P}$, there can be an additional suppression [40] which is not crucial for our subsequent discussion.
} 
in order to avoid a too small unified gauge coupling $g_{\mathrm{GUT}}^{2} \simeq 1 / \operatorname{Re}\left(\mathcal{F}_{A}\right)$. For $\Lambda_{\text {ins }} \gtrsim m_{3 / 2}$, the lower bound (2.28) or (2.30) and the upper bound (2.31) are numerically comparable to each other. This implies

$$
\tau=\mathcal{O}\left(\frac{8 \pi^{2}}{g_{\mathrm{GUT}}^{2}}\right)
$$

at least for light string-theoretic axions which can be identified as a QCD axion or an ALP constituting dark matter. Then, with such a large value of $\tau$, the following order of magnitude estimates are expected to be held:

$$
\begin{aligned}
\frac{\partial K_{0}}{\partial \tau} & \sim \tau \frac{\partial^{2} K_{0}}{\partial \tau^{2}} \sim \tau \frac{f_{a}^{2}}{M_{P}^{2}} \lesssim \mathcal{O}\left(\frac{g_{\mathrm{GUT}}^{2}}{8 \pi^{2}}\right), \\
\frac{\partial \ln Z_{I}}{\partial \tau} & \sim \frac{1}{\tau}=\mathcal{O}\left(\frac{g_{\mathrm{GUT}}^{2}}{8 \pi^{2}}\right),
\end{aligned}
$$

which suggest

$$
c_{\phi}\left(f_{a}\right)=\omega_{\phi} \frac{g_{\mathrm{GUT}}^{2}}{16 \pi^{2}}, \quad c_{\psi}\left(f_{a}\right)=\omega_{\psi} \frac{g_{\mathrm{GUT}}^{2}}{16 \pi^{2}}
$$

with

$$
\omega_{\phi} \sim \omega_{\psi}=\mathcal{O}(1) .
$$

Accordingly, the tree level couplings $C_{\Psi}^{0}(\Psi=u, d, e)$ of string-theoretic axions to the light quarks and electron, which are defined in (2.5), are estimated as

$$
\text { String-theoretic : } \quad C_{\Psi}^{0}=\mathcal{O}\left(\frac{g_{\mathrm{GUT}}^{2}}{16 \pi^{2}}\right) \quad(\Psi=u, d, e) .
$$

Note that this estimate applies for string-theoretic axions which have a non-zero coupling to at least one of the SM gauge fields and also light enough to be identified as a QCD axion or an ALP dark matter.

The above estimate indicates that the couplings of string-theoretic axions have a similar pattern as those of KSVZ-like axions in the sense that both axions have $\left|C_{\Psi}^{0} / c_{A}\right| \ll 1$. Yet one might be able to distinguish one from the other if the radiative corrections to $C_{\Psi}$ are significantly smaller than the tree-level couplings $C_{\Psi}^{0}$ for string-theoretic axions. In such case, the low energy couplings $C_{\Psi}$ of KSVZ-like axions will be significantly smaller than those of string-theoretic axions, which would leave a notable imprint in the observable axion couplings $g_{a X}(X=\gamma, p, n, e)$ in (2.7). To see if KSVZ-like axions and string-theoretic axions indeed have a distinguishable pattern of $g_{a X}$, we will conduct in section 3 an RG analysis for the axion couplings to matter fields.

In fact, the estimate (2.34) can be elaborated with the scaling argument made in [48]. For gauge fields propagating over a $d_{\mathrm{YM}}$-dimensional cycle $\Sigma_{\mathrm{YM}}$ in 6-dimensional internal space, ${ }^{3}$ the corresponding gauge couplings scale as

$$
\operatorname{Re}\left(\mathcal{F}_{A}\right)=\frac{1}{g_{A}^{2}} \propto \mathcal{V}\left(\Sigma_{\mathrm{YM}}\right)
$$

\footnotetext{
${ }^{3}$ In heterotic string theory, gauge fields propagate over the entire 6-dimensional internal space, so $d_{\mathrm{YM}}=6$. In other string theories with $D$-branes, gauge fields are confined on $D_{p}$-branes wrapping a $(p-3)$-dimensional cycle, so $d_{\mathrm{YM}}=p-3$.
} 
at leading order in $1 / \mathcal{V}\left(\Sigma_{\mathrm{YM}}\right)$, which ignores for instance the flux densities in $\Sigma_{\mathrm{YM}}$, where $\mathcal{V}\left(\Sigma_{\mathrm{YM}}\right)$ is the volume (in the string frame) of $\Sigma_{\mathrm{YM}}$. On the other hand, the physical Yukawa couplings $y_{I J K}$ are determined as

$$
y_{I J K}=\int_{\Sigma_{I J K}} \phi_{I}^{(0)} \phi_{J}^{(0)} \phi_{K}^{(0)} d \mathcal{V}\left(\Sigma_{I J K}\right),
$$

where $\phi_{I}^{(0)}$ is the zero mode wavefunction of $\Phi^{I}$ which can propagate over a $d_{I}$-dimensional subcycle $\Sigma_{I}$ of $\Sigma_{\mathrm{YM}}$, and $\Sigma_{I J K}$ is a $d_{I J K^{-}}$-dimensional subcycle over which the three zero mode wavefunctions $\phi_{I}^{(0)}, \phi_{J}^{(0)}$ and $\phi_{K}^{(0)}$ can have a nonzero overlap. Here $\phi_{I}^{(0)}$ is normalized as

$$
\int_{\Sigma_{I}}\left|\phi_{I}^{(0)}\right|^{2} d \mathcal{V}\left(\Sigma_{I}\right)=1
$$

Note that

$$
\Sigma_{\mathrm{YM}} \supseteq \Sigma_{I} \supseteq \Sigma_{I J K},
$$

and therefore

$$
d_{\mathrm{YM}} \geq d_{I} \geq d_{I J K}
$$

It has been argued in [48] that in the limit where $\mathcal{V}\left(\Sigma_{\mathrm{YM}}\right)$ is large enough and therefore the flux densities in $\Sigma_{\mathrm{YM}}$ can be ignored, the normalized zero mode wavefunction $\phi_{I}^{(0)}$ is simply rescaled as

$$
\phi_{I}^{(0)} \rightarrow \lambda^{-d_{I} / 2 d_{\mathrm{YM}}} \phi_{I}^{(0)}
$$

under the rescaling

$$
\mathcal{V}\left(\Sigma_{\mathrm{YM}}\right) \rightarrow \lambda \mathcal{V}\left(\Sigma_{\mathrm{YM}}\right) .
$$

Then the associated Yukawa couplings are rescaled as

$$
y_{I J K} \rightarrow \lambda^{\left(2 d_{I J K}-d_{I}-d_{J}-d_{K}\right) / 2 d_{\mathrm{YM}}} y_{I J K} .
$$

On the other hand, the physical Yukawa couplings in 4-dimensional supergravity are given by

$$
y_{I J K}=\frac{\lambda_{I J K}}{\sqrt{Y_{I} Y_{J} Y_{K}}},
$$

where $\lambda_{I J K}$ are the holomorphic Yukawa couplings in the superpotential:

$$
W_{\text {Yukawa }}=\frac{1}{6} \lambda_{I J K} \Phi^{I} \Phi^{J} \Phi^{K}
$$

and

$$
Y_{I}=e^{-K_{0} / 3} Z_{I}
$$

Due to the holomorphicity and the U(1) $)_{\mathrm{PQ}}$ symmetry, $\lambda_{I J K}$ are independent of $\mathcal{V}\left(\Sigma_{\mathrm{YM}}\right)$. Since $\operatorname{Re}\left(\mathcal{F}_{A}\right)=1 / g_{A}^{2} \propto \mathcal{V}\left(\Sigma_{\mathrm{YM}}\right),(2.44)$ and (2.45) suggest

$$
Y_{I}=e^{-K_{0} / 3} Z_{I} \propto\left(\operatorname{Re}\left(\mathcal{F}_{A}\right)\right)^{\omega_{I}},
$$

where the scaling weights $\omega_{I}$ are rational numbers constrained as

$$
\omega_{I}+\omega_{J}+\omega_{K}=\frac{1}{d_{\mathrm{YM}}}\left(d_{I}+d_{J}+d_{K}-2 d_{I J K}\right)
$$


for the combinations having a nonzero Yukawa coupling. This implies

$$
\frac{\partial \ln Y_{I}}{\partial T} \simeq \omega_{I} \frac{\partial \ln \operatorname{Re}\left(\mathcal{F}_{A}\right)}{\partial T}=\omega_{I} \frac{c_{A} g_{A}^{2}}{16 \pi^{2}}
$$

which confirms the estimates (2.33) and (2.34). Although the above scaling argument is valid only at leading order in $1 / \mathcal{V}\left(\Sigma_{\mathrm{YM}}\right)$, it is likely to give a correct order of magnitude estimate even when higher order corrections become non-negligible.

An interesting string model which can incorporate a QCD axion is the Large Volume Scenario (LVS) [49] of Type IIB string compactification on a Calabi-Yau (CY) space which involves a 4-cycle wrapped by $D 7$ branes supporting the SM gage fields. It has been argued that in this scenario all moduli including the saxion partner of the QCD axion can be successfully stabilized with $f_{a}$ exponentially lower than the Planck scale [40, 44]. At leading order approximation to ignore the effects of magnetic fluxes on $D 7$ branes, the Kahler potential and the holomorphic gauge kinetic function of the model are given by $[40,48]$

$$
K=-3 \ln \left(T_{b}+T_{b}^{*}\right)+\frac{\left(T+T^{*}\right)^{3 / 2}}{\left(T_{b}+T_{b}^{*}\right)^{3 / 2}}+\frac{\left(T+T^{*}\right)^{\omega_{I}}}{\left(T_{b}+T_{b}^{*}\right)} \Phi_{I}^{*} \Phi_{I}, \quad \mathcal{F}_{A}=\frac{1}{8 \pi^{2}} T
$$

where

$$
T_{b}=\tau_{b}+i \theta_{b}, \quad T=\tau+i \frac{a}{f_{a}}
$$

and the scaling weights $\omega_{I}$ are rational numbers between 0 and 1 as suggested by (2.49). Note that the factor $1 / 8 \pi^{2}$ in $\mathcal{F}_{A}$ is due to the normalization convention of $\operatorname{Im}(T)=$ $a(x) / f_{a} \cong \operatorname{Im}(T)+2 \pi$, where $a(x)$ is the QCD axion in the model. Here the Kahler modulus $\tau_{b}$ corresponds to the volume of an exponentially big 4-cycle in CY space, with which the 4-dimensional Planck scale $M_{P}$ can be exponentially bigger than the string scale $M_{\mathrm{st}}$ as

$$
\tau_{b} \sim \mathcal{V}_{\mathrm{CY}}^{2 / 3} \sim\left(\frac{M_{P}}{M_{\mathrm{st}}}\right)^{4 / 3} \gg \frac{8 \pi^{2}}{g_{\mathrm{GUT}}^{2}}
$$

where $\mathcal{V}_{\mathrm{CY}}$ is the $\mathrm{CY}$ volume, while $\tau$ describes the volume of a moderately large 4-cycle wrapped by $D 7$ branes supporting the SM gauge fields, which determines the SM gauge couplings at the string scale as

$$
\tau=\frac{8 \pi^{2}}{g_{\mathrm{GUT}}^{2}} .
$$

For simplicity, in (2.51) we ignored the part depending on other moduli while keeping only the part relevant for the couplings and the decay constant of the QCD axion $a(x)$. We then find

$$
f_{a}=\frac{\sqrt{3}}{2 \sqrt{2}} \frac{M_{P}}{\tau^{1 / 4} \tau_{b}^{3 / 4}}
$$

together with the following couplings to gauge and matter fields:

$$
c_{A}=1, \quad c_{\phi_{I}}\left(f_{a}\right)=\omega_{I} \frac{g_{\mathrm{GUT}}^{2}}{16 \pi^{2}}+\mathcal{O}\left(\frac{1}{\tau_{b}}\right), \quad c_{\psi_{I}}=\omega_{I} \frac{g_{\mathrm{GUT}}^{2}}{16 \pi^{2}}+\mathcal{O}\left(\frac{1}{\tau_{b}}\right) .
$$


This model of string-theoretic axion is particularly interesting as it can give rise to an axion decay constant anywhere between $f_{a} \sim 10^{16} \mathrm{GeV}$ (for $\tau_{b} \sim \tau$ ) and the observational lower bound $f_{a} \sim 10^{9} \mathrm{GeV}$ (for an exponentially large $\tau_{b} \sim 10^{11} \gg \tau$ ). Therefore, in section 4, we use this model as a benchmark model for string-theoretic axion and compare its low energy couplings $g_{a X}(X=\gamma, p, n, e)$ with those of KSVZ-like axions. More specifically, we will consider a model with chiral matters on the intersections of $D 7$ branes, for which $d_{\mathrm{YM}}=4, d_{I}=2$ and $d_{I J K}=0$, and therefore [48]

$$
\omega_{I}=\frac{1}{2} .
$$

In order to see that the order of magnitude estimate (2.34) is valid even when the flux densities in $\Sigma_{\mathrm{YM}}$ are not negligible, let us consider another example of string-theoretic axions, the model-independent axion and the model-dependent Kahler axion in heterotic string or $M$-theory in which the underlying YM and gravitational fluxes play a key role for the model to accommodate a QCD axion [50-53]. For simplicity, we consider a compactification on CY space with a single Kahler axion, which involves

$$
S=\tau_{1}+i \frac{a_{1}}{f_{1}}, \quad T=\tau_{2}+i \frac{a_{2}}{f_{2}},
$$

where $a_{1} \cong a_{1}+2 \pi f_{1}$ and $a_{2} \cong a_{2}+2 \pi f_{2}$ are the model-independent axion and the Kahler axion, respectively, that originate from the NS 2-form field $B$ as

$$
\partial_{\mu} a_{1} \propto \epsilon_{\mu \nu \rho \sigma} \partial^{\nu} B^{\rho \sigma}, \quad a_{2} \propto \int_{\Sigma} B_{i \bar{j}} d z^{i} \wedge d z^{* j},
$$

where $\Sigma$ is the 2 -cycle dual to the Kahler form. In the heterotic string regime, $\tau_{1}$ correspond to the Euclidean action of the NS5 brane instanton wrapping the entire CY space [39], and $\tau_{2}$ is that of the world-sheet instanton wrapping $\Sigma[46]$ :

$$
\tau_{1} \propto \frac{\mathcal{V}_{\mathrm{CY}}}{g_{\mathrm{st}}^{2}}, \quad \tau_{2} \propto \mathcal{V}(\Sigma),
$$

where $g_{\text {st }}$ is the string coupling. The moduli $\tau_{1}$ and $\tau_{2}$ can be extrapolated to the heterotic $M$-theory regime [54] where they correspond to the Euclidean actions of the $M 5$ brane instanton wrapping the CY space and the $M 2$ brane instanton wrapping $\Sigma$ stretched along the 11-th dimension.

The moduli Kahler potential, matter Kahler metric, and holomorphic gauge kinetic functions of the above model are given by [55]

$$
\begin{aligned}
K_{0} & =-\ln \left(S+S^{*}\right)-3 \ln \left(T+T^{*}\right) \\
Z_{I} & =\frac{1}{T+T^{*}}-\frac{\epsilon}{3} \frac{1}{S+S^{*}}, \\
\mathcal{F}_{E 6} & =\frac{1}{8 \pi^{2}}(S+\epsilon T), \quad \mathcal{F}_{E 8}=\frac{1}{8 \pi^{2}}(S-\epsilon T)
\end{aligned}
$$

where $\mathcal{F}_{E 6}$ and $\mathcal{F}_{E 8}$ are the gauge kinetic functions of the visible $E_{6}$ and the hidden $E_{8}$, respectively, and

$$
\epsilon=\frac{1}{8 \pi^{2}} \int J \wedge\left[\operatorname{tr}(F \wedge F)-\frac{1}{2} \operatorname{tr}(R \wedge R)\right]
$$


for the Kahler form $J$. Obviously the pieces proportional to $\epsilon$ are the corrections induced by flux density, which can be interpreted as string loop corrections in the heterotic string regime $[51,56,57]$, or as a flux-induced warping of CY geometry along the 11-th dimension in the heterotic $M$-theory regime [55, 58]. At leading order approximation which ignores these corrections, which would correspond to the weakly coupled heterotic string theory limit with $\epsilon \tau_{2} \ll \tau_{1}$, one finds

$$
Y_{I}=e^{-K_{0} / 3} Z_{I} \simeq\left(S+S^{*}\right)^{1 / 3} \propto\left(\operatorname{Re}\left(\mathcal{F}_{A}\right)\right)^{1 / 3}
$$

as suggested by the scaling relation (2.48) with $\omega_{I}=1 / 3$ which is implied by $(2.49)$ for gauge and matter fields propagating over the full CY space: $d_{\mathrm{YM}}=d_{I}=d_{I J K}=6$. However, due to the hidden $E_{8}$ gauge group confining at high scales as well as the world sheet instanton effects, a viable QCD axion can be obtained only in the heterotic $M$-theory limit with $\epsilon \tau_{2} \sim \tau_{1}=\mathcal{O}\left(8 \pi^{2} / g_{\text {GUT }}^{2}\right)[52,53]$. In such case, the QCD axion $a(x)$ corresponds to a combination of $a_{1}$ and $a_{2}$ which avoids the coupling to the hidden $E_{8}$ gauge fields. To find the decay constant and couplings of this QCD axion, one may parameterize the model-independent axion and the Kahler axion in terms of the QCD axion as

$$
\frac{a_{1}}{f_{1}} \propto \epsilon \frac{a}{f_{a}}, \quad \frac{a_{2}}{f_{2}} \propto \frac{a}{f_{a}},
$$

where the heavy axion component is frozen at its vacuum value by the potential induced by the hidden $E_{8}$ gauge fields. For the convenience of discussion, here we define $f_{a}$ as a scale for which the couplings of $a(x)$ to the $E_{6}$ gauge fields are given by $c_{A}=1$, not as a scale to define the periodicity of $a(x)$. It is then straightforward to find

$$
f_{a}=\sqrt{2} M_{P} \frac{g_{\text {GUT }}^{2}}{8 \pi^{2}} \frac{g_{\text {hid }}^{2} \sqrt{\left(g_{\text {hid }}^{2}+g_{\text {GUT }}^{2}\right)^{2}-g_{\text {hid }}^{2} g_{\text {GUT }}^{2}}}{\left|g_{\text {hid }}^{4}-g_{\text {GUT }}^{4}\right|}
$$

with the couplings

$$
c_{A}=1, \quad c_{\phi_{I}}\left(f_{a}\right)=\omega_{\phi} \frac{g_{\mathrm{GUT}}^{2}}{16 \pi^{2}}, \quad c_{\psi_{I}}=\omega_{\psi} \frac{g_{\mathrm{GUT}}^{2}}{16 \pi^{2}},
$$

where

$$
\begin{aligned}
& \omega_{\phi}=-\frac{g_{\text {hid }}^{2}\left[\left(g_{\text {hid }}^{2}+g_{\mathrm{GUT}}^{2}\right)^{2}+2 g_{\text {hid }}^{2} g_{\mathrm{GUT}}^{2}\right]}{\left(g_{\mathrm{hid}}^{2}+2 g_{\mathrm{GUT}}^{2}\right)\left(g_{\mathrm{hid}}^{4}-g_{\mathrm{GUT}}^{4}\right)}, \\
& \omega_{\psi}=\frac{g_{\text {hid }}^{2}\left[\left(g_{\text {hid }}^{2}+g_{\mathrm{GUT}}^{2}\right)^{2}-g_{\mathrm{hid}}^{2} g_{\mathrm{GUT}}^{2}\right]}{\left(g_{\text {hid }}^{2}+2 g_{\mathrm{GUT}}^{2}\right)\left(g_{\mathrm{hid}}^{4}-g_{\mathrm{GUT}}^{4}\right)}
\end{aligned}
$$

and $g_{\text {GUT }}$ and $g_{\text {hid }}$ are the gauge couplings around the string scale for the visible $E_{6}$ and the hidden $E_{8}$, respectively, which are determined by

$$
\operatorname{Re}\left(\mathcal{F}_{E_{6}}\right)=\frac{1}{g_{\text {GUT }}^{2}}, \quad \operatorname{Re}\left(\mathcal{F}_{E_{8}}\right)=\frac{1}{g_{\text {hid }}^{2}} .
$$

This result shows that the order of magnitude estimate (2.34) is valid even when the effects of flux densities are properly taken into account. 


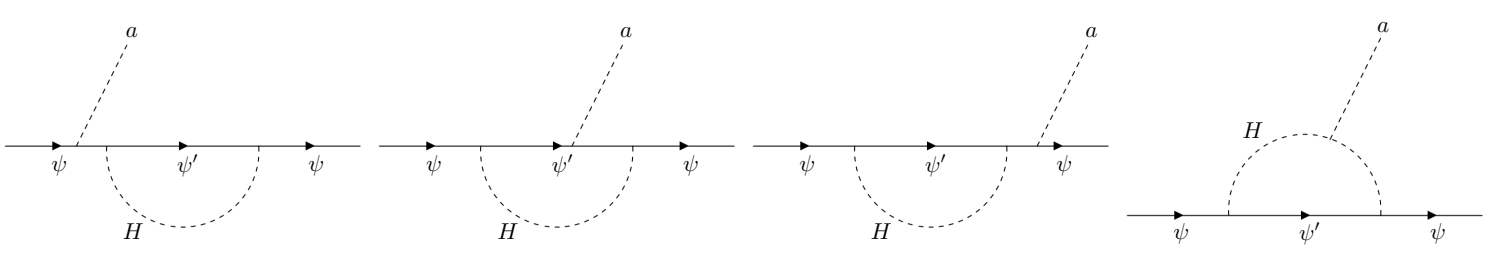

Figure 1. Diagrams for the Yukawa-induced renormalization group running of $\mathbf{c}_{\psi}$.

\section{Running of the axion couplings}

The low energy axion couplings can be substantially different from the UV boundary values discussed in the previous section because of the subsequent renormalization group evolution. In this section we will present the RG equations of axion couplings to the SM particles at leading order in dimensionless couplings (i.e. Yukawa and gauge couplings) in a generic way that can be applied to the SM, the minimal supersymmetric standard model (MSSM), and the two Higgs doublet models (2HDMs). We will then provide a semi-analytic formula for the solution of the RG equations as well as the numerical results.

\subsection{RG equations}

In the Georgi-Kaplan-Randall (GKR) field basis [26], the axion couplings to the SM fields and an additional Higgs doublet field in supersymmetric models or 2HDMs can be generally written

$$
\mathcal{L}_{a}=\frac{\partial_{\mu} a}{f_{a}}\left[\sum_{\psi}\left(\mathbf{c}_{\psi}\right)_{i j} \psi_{i}^{\dagger} \bar{\sigma}^{\mu} \psi_{j}+\sum_{\alpha=1,2} c_{H_{\alpha}} H_{\alpha}^{\dagger} i \stackrel{\leftrightarrow}{D^{\mu}} H_{\alpha}\right]+\frac{a}{f_{a}} \sum_{A} c_{A} \frac{g_{A}^{2}}{32 \pi^{2}} F^{A \mu \nu} \widetilde{F}_{\mu \nu}^{A},
$$

where $\psi_{i}=\left\{Q_{i}, u_{i}^{c}, d_{i}^{c}, L_{i}, e_{i}^{c}\right\}(i=1,2,3)$ are the left-handed quarks and leptons in the SM and $H_{\alpha}(\alpha=1,2)$ denote the two Higgs doublets in the model. The axion couplings to $\psi_{i}$ and $H_{\alpha}$ get renormalized by the Yukawa interactions involving $H_{\alpha}$ and $\psi_{i}$, which is determined at leading order by the diagrams in figure 1. For general 2HDMs, the Yukawa couplings can be written as

$$
\mathcal{L}_{\text {Yukawa }}=\sum_{f, F, \alpha, i, j}\left(\mathbf{y}_{f F \alpha}\right)_{i j} f_{i} F_{j} H_{\alpha},+ \text { h.c. }
$$

where $f_{i}=\left\{u_{i}^{c}, d_{i}^{c}, e_{i}^{c}\right\}$ are $\mathrm{SU}(2)_{L}$-singlet fermions, and $F_{i}=\left\{Q_{i}, L_{i}\right\}$ are $\mathrm{SU}(2)_{L}$-doublet fermions. The Yukawa-induced RG running of axion couplings at leading order are previously given in [21-25] for the SM or its supersymmetric extensions. Here we provide a general expression which can be applied to general 2HDMs as well:

$$
\begin{aligned}
\left.\frac{d \mathbf{c}_{F}}{d \ln \mu}\right|_{\text {Yukawa }} & =\frac{\xi_{y}}{16 \pi^{2}} \sum_{f, \alpha}\left(\frac{1}{2}\left\{\mathbf{c}_{F}, \mathbf{y}_{f F \alpha}^{\dagger} \mathbf{y}_{f F \alpha}\right\}+\mathbf{y}_{f F \alpha}^{\dagger} \mathbf{c}_{f}^{T} \mathbf{y}_{f F \alpha}+c_{H_{\alpha}} \mathbf{y}_{f F \alpha}^{\dagger} \mathbf{y}_{f F \alpha}\right), \\
\left.\frac{d \mathbf{c}_{f}^{T}}{d \ln \mu}\right|_{\text {Yukawa }} & =\frac{\xi_{y}}{16 \pi^{2}} \sum_{F, \alpha}\left(\frac{1}{2}\left\{\mathbf{c}_{f}^{T}, \mathbf{y}_{f F \alpha} \mathbf{y}_{f F \alpha}^{\dagger}\right\}+\mathbf{y}_{f F \alpha} \mathbf{c}_{F} \mathbf{y}_{f F \alpha}^{\dagger}+c_{H_{\alpha}} \mathbf{y}_{f F \alpha} \mathbf{y}_{f F \alpha}^{\dagger}\right), \\
\left.\frac{d c_{H_{\alpha}}}{d \ln \mu}\right|_{\text {Yukawa }} & =\frac{1}{8 \pi^{2}} \sum_{f, F}\left(c_{H_{\alpha}} \operatorname{tr}\left(\mathbf{y}_{f F \alpha}^{\dagger} \mathbf{y}_{f F \alpha}\right)+\operatorname{tr}\left(\mathbf{y}_{f F \alpha} \mathbf{c}_{F} \mathbf{y}_{f F \alpha}^{\dagger}\right)+\operatorname{tr}\left(\mathbf{y}_{f F \alpha}^{\dagger} \mathbf{c}_{f}^{T} \mathbf{y}_{f F \alpha}\right)\right),
\end{aligned}
$$



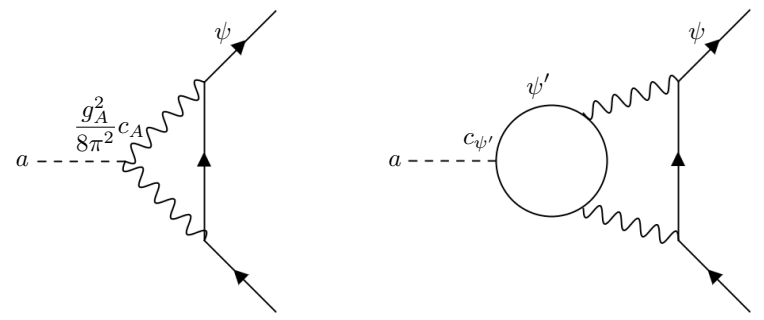

Figure 2. Diagrams for the gauge-induced renormalization group running of $\mathbf{c}_{\psi}$.

where

$$
\xi_{y}= \begin{cases}1 & \text { for non-SUSY models } \\ 2 & \text { for SUSY models }\end{cases}
$$

Gauge interactions also give rise to a running of axion couplings which is determined at leading order by the diagrams in figure 2. A general formula for such gauge-induced RG running of axion couplings is given in $[15,24,25]$ for the SM. Here we generalize the formula for SUSY models using a connection between the beta function of an axion coupling and the anomalous dimension of a chiral superfield as described in [21, 59]:

$$
\begin{aligned}
\left.\frac{d \mathbf{c}_{\psi}}{d \ln \mu}\right|_{\text {gauge }} & =-\xi_{g} \sum_{A} \frac{3}{2}\left(\frac{g_{A}^{2}}{8 \pi^{2}}\right)^{2} \mathbb{C}_{A}(\psi)\left(c_{A}-2 \sum_{\psi^{\prime}} \operatorname{tr}\left(\mathbf{c}_{\psi^{\prime}}\right) \mathbb{\mathbb { T }}_{A}\left(\psi^{\prime}\right)\right) \mathbb{1}, \\
\left.\frac{d c_{H_{\alpha}}}{d \ln \mu}\right|_{\text {gauge }} & =-\xi_{H} \sum_{A} \frac{3}{2}\left(\frac{g_{A}^{2}}{8 \pi^{2}}\right)^{2} \mathbb{C}_{A}\left(H_{\alpha}\right)\left(c_{A}-2 \sum_{\psi^{\prime}} \operatorname{tr}\left(\mathbf{c}_{\psi^{\prime}}\right) \mathbb{\mathbb { T }}_{A}\left(\psi^{\prime}\right)\right),
\end{aligned}
$$

where

$$
\xi_{g}=\left\{\begin{array}{ll}
1 & \text { for non-SUSY models } \\
2 / 3 & \text { for SUSY models }
\end{array}, \quad \xi_{H}= \begin{cases}0 & \text { for non-SUSY models } \\
2 / 3 & \text { for SUSY models }\end{cases}\right.
$$

The difference between non-SUSY models and SUSY modes is due to the presence of the axion-gaugino couplings in SUSY models. Here $\mathbb{C}_{A}(\Phi)$ and $\mathbb{T}_{A}(\Phi)$ are the quadratic Casimir and Dynkin index of the field $\Phi$ charged under the gauge group $A$.

In fact, eq. (3.3) and eq. (3.5) can be determined up to overall coefficients by the covariance of the RG equations under two spurious symmetries of the lagrangian. One of the symmetries is associated with the $\mathrm{SU}(3)$ flavor rotations of $\psi_{i}$ :

$$
\psi_{i} \rightarrow\left(U_{\psi}\right)_{i j} \psi_{j}, \quad \mathbf{c}_{\psi} \rightarrow U_{\psi} \mathbf{c}_{\psi} U_{\psi}^{\dagger}, \quad \mathbf{y}_{f F \alpha} \rightarrow U_{f}^{*} \mathbf{y}_{f F \alpha} U_{F}^{\dagger} .
$$

The other involves the following axion-dependent field redefinition which is allowed within the GKR field basis:

$$
\psi_{i} \rightarrow \psi_{i} e^{i x_{\psi_{i}} a / f_{a}}, \quad H_{\alpha} \rightarrow H_{\alpha} e^{i x_{H_{\alpha}} a / f_{a}}
$$


for $x_{\psi_{i}}$ and $x_{H_{\alpha}}$ satisfying

$$
\left(\mathbf{y}_{f F \alpha}\right)_{i j}\left(x_{f_{i}}+x_{F_{j}}+x_{H_{\alpha}}\right)=0
$$

with redefinition of parameters

$$
\left(\mathbf{c}_{\psi}\right)_{i j} \rightarrow\left(\mathbf{c}_{\psi}\right)_{i j}+x_{\psi_{i}} \delta_{i j}, \quad c_{H_{\alpha}} \rightarrow c_{H_{\alpha}}+x_{H_{\alpha}}, \quad c_{A} \rightarrow c_{A}+2 \sum_{\psi_{i}} x_{\psi_{i}} \mathbb{T}_{A}\left(\psi_{i}\right) .
$$

From the RG equations (3.3) and (3.5), we find that the condition for a non-trivial RG running of the couplings of field-theoretic axions can be expressed in terms of the linearly realized $\mathrm{U}(1)_{\mathrm{PQ}}$ symmetry. For field-theoretic axions, the axion couplings to matter fields in the GKR field basis at $\mu \sim f_{a}$ are identified as the PQ charges of those fields in the field basis where $\mathrm{U}(1)_{\mathrm{PQ}}$ is linearly realized as eq. (2.9), while the axion couplings to gauge fields in the GKR field basis is identified as the $\mathrm{U}(1)_{\mathrm{PQ}}$ anomaly for the gauge group. Then, from eq. (3.3), one can see that the RG running of axion couplings induced by a Yukawa coupling occurs when the sum of the PQ charges of the fields involved in the corresponding Yukawa coupling is non-vanishing. On the other hand, eq. (3.5) tells us that the RG running of axion couplings induced by gauge interaction starts from the scale where the heaviest PQand gauge-charged fermion is integrated out.

In the following analysis we will focus on the RG evolution of axion couplings in the MSSM and the SM. We write the Yukawa couplings in the MSSM as

$$
\mathcal{L}_{\text {Yukawa }}=\left(\tilde{\mathbf{y}}_{u}\right)_{i j} u_{i}^{c} Q_{j} H_{u}+\left(\tilde{\mathbf{y}}_{d}\right)_{i j} d_{i}^{c} Q_{j} H_{d}+\left(\tilde{\mathbf{y}}_{e}\right)_{i j} e_{i}^{c} L_{j} H_{d}+\text { h.c. }
$$

From the general formula eq. (3.3) and eq. (3.5), the full axion RG equations at leading order in the Yukawa and gauge couplings in the MSSM come out as

$$
\begin{aligned}
\frac{d \mathbf{c}_{Q}}{d \ln \mu}= & \frac{\xi_{y}}{16 \pi^{2}}\left(\frac{1}{2}\left\{\mathbf{c}_{Q}, \tilde{\mathbf{y}}_{u}^{\dagger} \tilde{\mathbf{y}}_{u}+\tilde{\mathbf{y}}_{d}^{\dagger} \tilde{\mathbf{y}}_{d}\right\}+\tilde{\mathbf{y}}_{u}^{\dagger} \mathbf{c}_{u c}^{T} \tilde{\mathbf{y}}_{u}+\tilde{\mathbf{y}}_{d}^{\dagger} \mathbf{c}_{d^{c}}^{T} \tilde{\mathbf{y}}_{d}+c_{H_{u}} \tilde{\mathbf{y}}_{u}^{\dagger} \tilde{\mathbf{y}}_{u}+c_{H_{d}} \tilde{\mathbf{y}}_{d}^{\dagger} \tilde{\mathbf{y}}_{d}\right) \\
& -\xi_{g}\left(\frac{\alpha_{s}^{2}}{2 \pi^{2}} \tilde{c}_{G}+\frac{9 \alpha_{2}^{2}}{32 \pi^{2}} \tilde{c}_{W}+\frac{\alpha_{1}^{2}}{96 \pi^{2}} \tilde{c}_{B}\right) \mathbb{1} \\
\frac{d \mathbf{c}_{u^{c}}^{T}}{d \ln \mu}= & \frac{\xi_{y}}{8 \pi^{2}}\left(\frac{1}{2}\left\{\mathbf{c}_{u c}^{T}, \tilde{\mathbf{y}}_{u} \tilde{\mathbf{y}}_{u}^{\dagger}\right\}+\tilde{\mathbf{y}}_{u} \mathbf{c}_{Q} \tilde{\mathbf{y}}_{u}^{\dagger}+c_{H_{u}} \tilde{\mathbf{y}}_{u} \tilde{\mathbf{y}}_{u}^{\dagger}\right)-\xi_{g}\left(\frac{\alpha_{s}^{2}}{2 \pi^{2}} \tilde{c}_{G}+\frac{\alpha_{1}^{2}}{6 \pi^{2}} \tilde{c}_{B}\right) \mathbb{1} \\
\frac{d \mathbf{c}_{d^{c}}^{T}}{d \ln \mu}= & \frac{\xi_{y}}{8 \pi^{2}}\left(\frac{1}{2}\left\{\mathbf{c}_{d^{c}}^{T}, \tilde{\mathbf{y}}_{d} \tilde{\mathbf{y}}_{d}^{\dagger}\right\}+\tilde{\mathbf{y}}_{d} \mathbf{c}_{Q} \tilde{\mathbf{y}}_{d}^{\dagger}+c_{H_{d}} \tilde{\mathbf{y}}_{d} \tilde{\mathbf{y}}_{d}^{\dagger}\right)-\xi_{g}\left(\frac{\alpha_{s}^{2}}{2 \pi^{2}} \tilde{c}_{G}+\frac{\alpha_{1}^{2}}{24 \pi^{2}} \tilde{c}_{B}\right) \mathbb{1} \\
\frac{d \mathbf{c}_{L}}{d \ln \mu}= & \frac{\xi_{y}}{16 \pi^{2}}\left(\frac{1}{2}\left\{\mathbf{c}_{L}, \tilde{\mathbf{y}}_{e}^{\dagger} \tilde{\mathbf{y}}_{e}\right\}+\tilde{\mathbf{y}}_{e}^{\dagger} \mathbf{c}_{e}^{T} \tilde{\mathbf{y}}_{e}+c_{H_{d}} \tilde{\mathbf{y}}_{e}^{\dagger} \tilde{\mathbf{y}}_{e}\right)-\xi_{g}\left(\frac{9 \alpha_{2}^{2}}{32 \pi^{2}} \tilde{c}_{W}+\frac{3 \alpha_{1}^{2}}{32 \pi^{2}} \tilde{c}_{B}\right) \mathbb{1} \\
\frac{d \mathbf{c}_{e^{c}}^{T}}{d \ln \mu}= & \frac{\xi_{y}}{8 \pi^{2}}\left(\frac{1}{2}\left\{\mathbf{c}_{e}^{T}, \tilde{\mathbf{y}}_{e} \tilde{\mathbf{y}}_{e}^{\dagger}\right\}+\tilde{\mathbf{y}}_{e} \mathbf{c}_{L} \tilde{\mathbf{y}}_{e}^{\dagger}+c_{H_{d}} \tilde{\mathbf{y}}_{e} \tilde{\mathbf{y}}_{e}^{\dagger}\right)-\xi_{g} \frac{3 \alpha_{1}^{2}}{8 \pi^{2}} \tilde{c}_{B} \mathbb{1} \\
\frac{d c_{H_{u}}}{d \ln \mu}= & \frac{3}{8 \pi^{2}}\left(c_{H_{u}} \operatorname{tr}\left(\tilde{\mathbf{y}}_{u}^{\dagger} \tilde{\mathbf{y}}_{u}\right)+\operatorname{tr}\left(\tilde{\mathbf{y}}_{u} \mathbf{c}_{Q} \tilde{\mathbf{y}}_{u}^{\dagger}\right)+\operatorname{tr}\left(\tilde{\mathbf{y}}_{u}^{\dagger} \mathbf{c}_{u^{c}}^{T} \tilde{\mathbf{y}}_{u}\right)\right)-\xi_{H}\left(\frac{9 \alpha_{2}^{2}}{32 \pi^{2}} \tilde{c}_{W}+\frac{3 \alpha_{1}^{2}}{32 \pi^{2}} \tilde{c}_{B}\right) \\
\frac{d c_{H_{d}}}{d \ln \mu}= & \frac{3}{8 \pi^{2}}\left(c_{H_{d}} \operatorname{tr}\left(\tilde{\mathbf{y}}_{d}^{\dagger} \tilde{\mathbf{y}}_{d}\right)+\operatorname{tr}\left(\tilde{\mathbf{y}}_{d} \mathbf{c}_{Q} \tilde{\mathbf{y}}_{d}^{\dagger}\right)+\operatorname{tr}\left(\tilde{\mathbf{y}}_{d}^{\dagger} \mathbf{c}_{d c}^{T} \tilde{\mathbf{y}}_{d}\right)\right) \\
& +\frac{1}{8 \pi^{2}}\left(c_{H_{d}} \operatorname{tr}\left(\tilde{\mathbf{y}}_{e}^{\dagger} \tilde{\mathbf{y}}_{e}\right)+\operatorname{tr}\left(\tilde{\mathbf{y}}_{e} \mathbf{c}_{L} \tilde{\mathbf{y}}_{e}^{\dagger}\right)+\operatorname{tr}\left(\tilde{\mathbf{y}}_{e}^{\dagger} \mathbf{c}_{e}^{T} \tilde{\mathbf{y}}_{e}\right)\right)-\xi_{H}\left(\frac{9 \alpha_{2}^{2}}{32 \pi^{2}} \tilde{c}_{W}+\frac{3 \alpha_{1}^{2}}{32 \pi^{2}} \tilde{c}_{B}\right)
\end{aligned}
$$


with

$$
\begin{aligned}
& \tilde{c}_{G}=c_{G}-\operatorname{tr}\left(2 \mathbf{c}_{\mathrm{Q}}+\mathbf{c}_{\mathrm{u}^{\mathrm{c}}}+\mathbf{c}_{\mathrm{d}^{\mathrm{c}}}\right), \\
& \tilde{c}_{W}=c_{W}-\operatorname{tr}\left(3 \mathbf{c}_{\mathrm{Q}}+\mathbf{c}_{\mathrm{L}}\right)-\frac{3}{2} \xi_{H}\left(c_{\widetilde{H}_{u}}+c_{\widetilde{H}_{d}}\right), \\
& \tilde{c}_{B}=c_{B}-\operatorname{tr}\left(\frac{1}{3}\left(\mathbf{c}_{\mathrm{Q}}+8 \mathbf{c}_{\mathrm{u}^{\mathrm{c}}}+2 \mathbf{c}_{\mathrm{d}^{\mathrm{c}}}\right)+\mathbf{c}_{\mathrm{L}}+2 \mathbf{c}_{\mathrm{e}^{\mathrm{c}}}\right)-\frac{3}{2} \xi_{H}\left(c_{\widetilde{H}_{u}}+c_{\widetilde{H}_{d}}\right) .
\end{aligned}
$$

The parameters $\tilde{c}_{G, W, B}$ in eq. (3.12) are invariant under the field redefinition (3.8). Here we note that $c_{\widetilde{H}_{u}}\left(=c_{H_{u}}\right)$ and $c_{\widetilde{H}_{d}}\left(=c_{H_{d}}\right)$ contributions in $\tilde{c}_{W}$ and $\tilde{c}_{B}$ are from the Higgsino loops.

In this work we are concerned with only the flavor diagonal components of the axion couplings. Therefore in the following we assume that $\left(\mathbf{c}_{\psi}\right)_{i j}$ are flavor diagonal in the mass eigenstate basis:

$$
\left(\mathbf{c}_{\psi}\right)_{i j}=c_{\psi_{i}} \delta_{i j}
$$

and approximate the Yukawa couplings in the RG equations by keeping only the third generation components:

$$
\left(\tilde{\mathbf{y}}_{u}\right)_{i j} \approx \tilde{y}_{t} \delta_{i 3} \delta_{j 3}, \quad\left(\tilde{\mathbf{y}}_{d}\right)_{i j} \approx \tilde{y}_{b} \delta_{i 3} \delta_{j 3}, \quad\left(\tilde{\mathbf{y}}_{e}\right)_{i j} \approx \tilde{y}_{\tau} \delta_{i 3} \delta_{j 3} .
$$

In this approximation, the RG equations in eq. (3.11) can be rewritten as

$$
\begin{aligned}
\frac{d n_{u_{i}}}{d \ln \mu} \simeq & \frac{1}{16 \pi^{2}}\left(3\left(2+\xi_{y} \delta_{i 3}\right) \tilde{y}_{t}^{2} n_{t}+\xi_{y} \tilde{y}_{b}^{2} n_{b} \delta_{i 3}\right) \\
& -\left(\frac{\xi_{g}}{\pi^{2}} \alpha_{s}^{2} \tilde{c}_{G}+\frac{9\left(\xi_{g}+\xi_{H}\right)}{32 \pi^{2}} \alpha_{2}^{2} \tilde{c}_{W}+\frac{\left(17 \xi_{g}+9 \xi_{H}\right)}{96 \pi^{2}} \alpha_{1}^{2} \tilde{c}_{B}\right), \\
\frac{d n_{d_{i}}}{d \ln \mu} \simeq & \frac{1}{16 \pi^{2}}\left(\xi_{y} \tilde{y}_{t}^{2} n_{t} \delta_{i 3}+3\left(2+\xi_{y} \delta_{i 3}\right) \tilde{y}_{b}^{2} n_{b}+2 \tilde{y}_{\tau}^{2} n_{\tau}\right) \\
& -\left(\frac{\xi_{g}}{\pi^{2}} \alpha_{s}^{2} \tilde{c}_{G}+\frac{9\left(\xi_{g}+\xi_{H}\right)}{32 \pi^{2}} \alpha_{2}^{2} \tilde{c}_{W}+\frac{\left(5 \xi_{g}+9 \xi_{H}\right)}{96 \pi^{2}} \alpha_{1}^{2} \tilde{c}_{B}\right), \\
\frac{d n_{e_{i}}}{d \ln \mu} \simeq & \frac{1}{16 \pi^{2}}\left(6 \tilde{y}_{b}^{2} n_{b}+\left(2+3 \xi_{y} \delta_{i 3}\right) \tilde{y}_{\tau}^{2} n_{\tau}\right)-\left(\frac{9\left(\xi_{g}+\xi_{H}\right)}{32 \pi^{2}} \alpha_{2}^{2} \tilde{c}_{W}+\frac{3\left(5 \xi_{g}+\xi_{H}\right)}{32 \pi^{2}} \alpha_{1}^{2} \tilde{c}_{B}\right), \\
\frac{d n_{H}}{d \ln \mu} \simeq & \frac{1}{8 \pi^{2}}\left(3 \tilde{y}_{t}^{2} n_{t}+3 \tilde{y}_{b}^{2} n_{b}+\tilde{y}_{\tau}^{2} n_{\tau}\right)-\xi_{H}\left(\frac{9}{16 \pi^{2}} \alpha_{2}^{2} \tilde{c}_{W}+\frac{3}{16 \pi^{2}} \alpha_{1}^{2} \tilde{c}_{B}\right) .
\end{aligned}
$$

where $u_{i}=(u, c, t), d_{i}=(d, s, b), e_{i}=(e, \mu, \tau)$, and

$$
\begin{aligned}
n_{u_{i}} \equiv c_{Q_{i}}+c_{u_{i}^{c}}+c_{H_{u}}, & n_{d_{i}} \equiv c_{n} Q_{i}+c_{d_{i}^{c}}+c_{H_{d}}, \\
n_{e_{i}} \equiv c_{L_{i}}+c_{e_{i}^{c}}+c_{H_{d}}, & n_{H} \equiv c_{H_{u}}+c_{H_{d}} .
\end{aligned}
$$

Note that the parameters defined in eq. (3.16) are invariant under the field redefinition (3.8), so they may be directly related to physically observable quantities as we will see below. In eq. (3.15), the RG coefficients $\tilde{c}_{G}, \tilde{c}_{W}$ and $\tilde{c}_{B}$ are scale-dependent because the axion-fermion couplings in eq. (3.12) are running, while

$$
\frac{d c_{A}}{d \ln \mu}=0 \quad(A=G, W, B) .
$$


From eq. (3.17) and eq. (3.11), one can derive

$$
\begin{aligned}
\frac{d \tilde{c}_{G}}{d \ln \mu} \simeq & -\frac{\xi_{y}}{4 \pi^{2}}\left(\tilde{y}_{t}^{2} n_{t}+\tilde{y}_{b}^{2} n_{b}\right)+\xi_{g}\left(\frac{6 \alpha_{s}^{2}}{\pi^{2}} \tilde{c}_{G}+\frac{27 \alpha_{2}^{2}}{16 \pi^{2}} \tilde{c}_{W}+\frac{11 \alpha_{1}^{2}}{16 \pi^{2}} \tilde{c}_{B}\right), \\
\frac{d \tilde{c}_{W}}{d \ln \mu} \simeq & -\frac{\left(\xi_{y}+3 \xi_{H}\right)}{16 \pi^{2}}\left(3 \tilde{y}_{t}^{2} n_{t}+3 \tilde{y}_{b}^{2} n_{b}+\tilde{y}_{\tau}^{2} n_{\tau}\right) \\
& +\frac{9 \xi_{g}}{2 \pi^{2}} \alpha_{s}^{2} \tilde{c}_{G}+\frac{\left(108 \xi_{g}+27 \xi_{H}^{2}\right)}{32 \pi^{2}} \alpha_{2}^{2} \tilde{c}_{W}+\frac{\left(12 \xi_{g}+9 \xi_{H}^{2}\right)}{32 \pi^{2}} \alpha_{1}^{2} \tilde{c}_{B}, \\
\frac{d \tilde{c}_{B}}{d \ln \mu} \simeq & -\frac{\left(17 \xi_{y}+27 \xi_{H}\right)}{48 \pi^{2}} \tilde{y}_{t}^{2} n_{t}-\frac{\left(5 \xi_{y}+27 \xi_{H}\right)}{48 \pi^{2}} \tilde{y}_{b}^{2} n_{b}-\frac{\left(5 \xi_{y}+3 \xi_{H}\right)}{16 \pi^{2}} \tilde{y}_{\tau}^{2} n_{\tau} \\
& +\frac{11 \xi_{g}}{2 \pi^{2}} \alpha_{s}^{2} \tilde{c}_{G}+\frac{\left(36 \xi_{g}+27 \xi_{H}^{2}\right)}{32 \pi^{2}} \alpha_{2}^{2} \tilde{c}_{W}+\frac{\left(380 \xi_{g}+27 \xi_{H}^{2}\right)}{96 \pi^{2}} \alpha_{1}^{2} \tilde{c}_{B} .
\end{aligned}
$$

Eq. (3.15) and eq. (3.18) constitute a complete set of RG equations given in terms of the $\mathrm{RG}$ parameters invariant under the field redefinition (3.8).

The above RG equations are valid when the RG scale $\mu$ is higher than mass scales of new particles beyond the standard model (BSM). For simplicity, let us assume that the masses of the superparticles and charged Higgs boson mass in 2HDMs are given by a common mass scale $m_{\mathrm{BSM}}$,

$$
m_{\mathrm{BSM}} \sim m_{\mathrm{SUSY}} \sim m_{H^{ \pm}}
$$

Below the BSM scale $\left(\mu<m_{\mathrm{BSM}}\right)$, the BSM particles are integrated out and no longer contribute to the running of the axion couplings. We will assume that $m_{\mathrm{BSM}}$ is well above the weak scale so that the light Higgs doublet field is approximated as the SM Higgs field with negligible mixing effect. Thus the RG equations are governed by the SM degrees of freedom including the SM Higgs field $H$. As the BSM Higgs doublet field is integrated out, the SM Yukawa couplings $y_{t}, y_{b}$ and $y_{\tau}$ satisfy the matching condition

$$
y_{t}=\tilde{y}_{t} \sin \beta, \quad y_{b}=\tilde{y}_{b} \cos \beta, \quad y_{\tau}=\tilde{y}_{\tau} \cos \beta \quad \text { at } \mu=m_{\mathrm{BSM}}
$$

where $\tan \beta \equiv\left\langle H_{u}\right\rangle /\left\langle H_{d}\right\rangle$, while the coupling between the axion and the SM Higgs field $c_{H}$ satisfies

$$
c_{H}=c_{H_{u}} \sin ^{2} \beta-c_{H_{d}} \cos ^{2} \beta \quad \text { at } \mu=m_{\mathrm{BSM}} .
$$

Then the RG equations for the axion couplings below the BSM scale (but above the weak 
scale) turn out to be

$$
\begin{aligned}
\frac{d c_{Q_{i}}}{d \ln \mu} & \simeq \frac{1}{16 \pi^{2}} y_{t}^{2}\left(c_{Q_{3}}+c_{u_{3}^{c}}+c_{H}\right) \delta_{i 3}-\left(\frac{\alpha_{s}^{2}}{2 \pi^{2}} \tilde{c}_{G}+\frac{9 \alpha_{2}^{2}}{32 \pi^{2}} \tilde{c}_{W}+\frac{\alpha_{1}^{2}}{96 \pi^{2}} \tilde{c}_{B}\right), \\
\frac{d c_{u_{i}^{c}}}{d \ln \mu} & \simeq \frac{1}{8 \pi^{2}} y_{t}^{2}\left(c_{Q_{3}}+c_{u_{3}^{c}}+c_{H}\right) \delta_{i 3}-\left(\frac{\alpha_{s}^{2}}{2 \pi^{2}} \tilde{c}_{G}+\frac{\alpha_{1}^{2}}{6 \pi^{2}} \tilde{c}_{B}\right), \\
\frac{d c_{d_{i}^{c}}}{d \ln \mu} & \simeq-\left(\frac{\alpha_{s}^{2}}{2 \pi^{2}} \tilde{c}_{G}+\frac{\alpha_{1}^{2}}{24 \pi^{2}} \tilde{c}_{B}\right), \\
\frac{d c_{L_{i}}}{d \ln \mu} & \simeq-\left(\frac{9 \alpha_{2}^{2}}{32 \pi^{2}} \tilde{c}_{W}+\frac{3 \alpha_{1}^{2}}{32 \pi^{2}} \tilde{c}_{B}\right), \\
\frac{d c_{e_{i}^{c}}}{d \ln \mu} & \simeq-\frac{3 \alpha_{1}^{2}}{8 \pi^{2}} \tilde{c}_{B} \\
\frac{d c_{H}}{d \ln \mu} & \simeq \frac{3}{8 \pi^{2}} y_{t}^{2}\left(c_{Q_{3}}+c_{u_{3}^{c}}+c_{H}\right),
\end{aligned}
$$

where we ignore the contributions from the bottom and tau Yukawa couplings $y_{b}, y_{\tau}$, and

$$
\begin{aligned}
& \tilde{c}_{G}=c_{G}-\sum_{i}\left(2 c_{Q_{i}}+c_{u_{i}^{c}}+c_{d_{i}^{c}}\right), \\
& \tilde{c}_{W}=c_{W}-\sum_{i}\left(3 c_{Q_{i}}+c_{L_{i}}\right), \\
& \tilde{c}_{B}=c_{B}-\sum_{i}\left(\frac{1}{3}\left(c_{Q_{i}}+8 c_{u_{i}^{c}}+2 c_{d_{i}^{c}}\right)+c_{L_{i}}+2 c_{e_{i}^{c}}\right) .
\end{aligned}
$$

Note that there are no longer Higgsino contributions in $\tilde{c}_{W, B}$ of eq. (3.23) compared to eq. (3.12). In fact the RG equations (3.22) can be recast into a simpler form:

$$
\begin{aligned}
& \frac{d C_{u_{i}}}{d \ln \mu} \simeq \frac{3}{16 \pi^{2}} y_{t}^{2} C_{t}\left(2+\delta_{i 3}\right)-\left(\frac{\alpha_{s}^{2}}{\pi^{2}} \tilde{c}_{G}+\frac{9 \alpha_{2}^{2}}{32 \pi^{2}} \tilde{c}_{W}+\frac{17 \alpha_{1}^{2}}{96 \pi^{2}} \tilde{c}_{B}\right), \\
& \frac{d C_{d_{i}}}{d \ln \mu} \simeq-\frac{1}{16 \pi^{2}} y_{t}^{2} C_{t}\left(6-\delta_{i 3}\right)-\left(\frac{\alpha_{s}^{2}}{\pi^{2}} \tilde{c}_{G}+\frac{9 \alpha_{2}^{2}}{32 \pi^{2}} \tilde{c}_{W}+\frac{5 \alpha_{1}^{2}}{96 \pi^{2}} \tilde{c}_{B}\right), \\
& \frac{d C_{e_{i}}}{d \ln \mu} \simeq-\frac{3}{8 \pi^{2}} y_{t}^{2} C_{t}-\left(\frac{9 \alpha_{2}^{2}}{32 \pi^{2}} \tilde{c}_{W}+\frac{15 \alpha_{1}^{2}}{32 \pi^{2}} \tilde{c}_{B}\right) .
\end{aligned}
$$

where

$$
\begin{aligned}
C_{u_{i}} & \equiv c_{Q_{i}}+c_{u_{i}^{c}}+c_{H}=n_{u_{i}}-n_{H} \cos ^{2} \beta, \\
C_{d_{i}} & \equiv c_{Q_{i}}+c_{d_{i}^{c}}-c_{H}=n_{d_{i}}-n_{H} \sin ^{2} \beta, \\
C_{e_{i}} & \equiv c_{L_{i}}+c_{e_{i}^{c}}-c_{H}=n_{e_{i}}-n_{H} \sin ^{2} \beta .
\end{aligned}
$$

for $u_{i}=(u, c, t), d_{i}=(d, s, b)$ and $e_{i}=(e, \mu, \tau)$. We remark that the parameters defined in eq. (3.25) are invariant under the SM version of the field redefinition (3.8). Having 
eq. (3.24), a complete set of RG equations below the BSM scale includes the following equations for the running of $\tilde{c}_{A}(A=G, W, B)$.

$$
\begin{aligned}
\frac{d \tilde{c}_{G}}{d \ln \mu} & \simeq-\frac{1}{4 \pi^{2}} y_{t}^{2} C_{t}+\frac{6 \alpha_{s}^{2}}{\pi^{2}} \tilde{c}_{G}+\frac{27 \alpha_{2}^{2}}{16 \pi^{2}} \tilde{c}_{W}+\frac{11 \alpha_{1}^{2}}{16 \pi^{2}} \tilde{c}_{B}, \\
\frac{d \tilde{c}_{W}}{d \ln \mu} & \simeq-\frac{3}{16 \pi^{2}} y_{t}^{2} C_{t}+\frac{9}{2 \pi^{2}} \alpha_{s}^{2} \tilde{c}_{G}+\frac{27}{8 \pi^{2}} \alpha_{2}^{2} \tilde{c}_{W}+\frac{3}{8 \pi^{2}} \alpha_{1}^{2} \tilde{c}_{B}, \\
\frac{d \tilde{c}_{B}}{d \ln \mu} & \simeq-\frac{17}{48 \pi^{2}} y_{t}^{2} C_{t}+\frac{11}{2 \pi^{2}} \alpha_{s}^{2} \tilde{c}_{G}+\frac{9}{8 \pi^{2}} \alpha_{2}^{2} \tilde{c}_{W}+\frac{95}{24 \pi^{2}} \alpha_{1}^{2} \tilde{c}_{B} .
\end{aligned}
$$

Below the weak scale $\left(\mu<m_{W}\right)$, the top quark, Higgs boson, and $W / Z$ gauge bosons are integrated out. We ignore the corresponding threshold corrections calculated in [25] because they are of subleading order giving a correction to our calculation less than $10 \%$. At this scale, the relevant axion couplings take the form

$$
c_{\gamma} \frac{e^{2}}{32 \pi^{2}} \frac{a}{f_{a}} F^{\mu \nu} \tilde{F}_{\mu \nu}+\frac{\partial_{\mu} a}{2 f_{a}}\left[C_{u_{i}} \bar{u}_{i} \gamma^{\mu} \gamma_{5} u_{i}+C_{d_{i}} \bar{d}_{i} \gamma^{\mu} \gamma_{5} d_{i}+C_{e_{i}} \bar{e}_{i} \gamma^{\mu} \gamma_{5} e_{i}\right]
$$

where $F^{\mu \nu}$ is the electromagnetic field strength, and $u_{i}, d_{i}$ and $e_{i}$ denote the Dirac quarks and leptons with masses lighter than the RG point $\mu$. Here

$$
c_{\gamma}=c_{W}+c_{B}
$$

and the matching conditions for $C_{u_{i}}, C_{d_{i}}$ and $C_{e_{i}}$ at the weak scale are given in eq. (3.25). The RG equations for the above axion couplings are given by

$$
\begin{aligned}
\frac{d C_{u_{i}}}{d \ln \mu} & \simeq-\frac{\alpha_{s}^{2}}{\pi^{2}} \tilde{c}_{G}-\frac{\alpha_{\mathrm{em}}^{2}}{6 \pi^{2}} \tilde{c}_{\gamma}, \\
\frac{d C_{d_{i}}}{d \ln \mu} & \simeq-\frac{\alpha_{s}^{2}}{\pi^{2}} \tilde{c}_{G}-\frac{\alpha_{\mathrm{em}}^{2}}{24 \pi^{2}} \tilde{c}_{\gamma}, \\
\frac{d C_{e_{i}}}{d \ln \mu} & \simeq-\frac{3 \alpha_{\mathrm{em}}^{2}}{8 \pi^{2}} \tilde{c}_{\gamma},
\end{aligned}
$$

where

$$
\begin{aligned}
& \tilde{c}_{G}(\mu)=c_{G}-\sum_{q} C_{q}(\mu) \Theta\left(\mu-m_{q}\right), \\
& \tilde{c}_{\gamma}(\mu)=c_{\gamma}-2 \sum_{f} N_{c}^{f} Q_{f}^{2} C_{f}(\mu) \Theta\left(\mu-m_{f}\right),
\end{aligned}
$$

with the unit step function $\Theta(x)=1$ for $x>0$ and $\Theta(x)=0$ for $x<0$. Here $q$ stands for the quarks $u, d, s, c, b$, and $N_{c}^{f}$ and $Q_{f}$ are the number of colors and the electromagnetic charge of the SM fermion $f$ lighter than the top quark, respectively.

Finally below the QCD scale $(\mu \lesssim 1 \mathrm{GeV})$, the relevant axion couplings can be written as

$$
\frac{1}{4} g_{a \gamma} a \vec{E} \cdot \vec{B}+\partial_{\mu} a\left[\frac{g_{a e}}{2 m_{e}} \bar{e} \gamma^{\mu} \gamma_{5} e+\frac{g_{a n}}{2 m_{n}} \bar{n} \gamma^{\mu} \gamma_{5} n+\frac{g_{a p}}{2 m_{p}} \bar{p} \gamma^{\mu} \gamma_{5} p\right]
$$


where the axion-photon coupling $g_{a \gamma}$ includes the threshold correction from the axion-pion mixing:

$$
g_{a \gamma} \simeq \frac{\alpha_{\mathrm{em}}}{2 \pi} \frac{1}{f_{a}}\left(c_{\gamma}-\frac{2}{3} \frac{m_{u}+4 m_{d}}{m_{u}+m_{d}} c_{G}\right) \simeq \frac{\alpha_{\mathrm{em}}}{2 \pi} \frac{1}{f_{a}}\left(c_{\gamma}-1.92 c_{G}\right),
$$

the axion-electron coupling $g_{a e}$ evolves down to $\mu=m_{e}$ as

$$
\frac{d g_{a e}}{d \ln \mu} \simeq-\frac{3 \alpha_{\mathrm{em}} m_{e}}{4 \pi} g_{a \gamma} \quad\left(g_{a e}=\frac{m_{e} C_{e}}{f_{a}}\right)
$$

and the axion-nucleon couplings can be computed from the couplings $c_{G}, C_{u}(\mu)$ and $C_{d}(\mu)$ at the matching scale $\mu=2 \mathrm{GeV}$ using the lattice data [27]:

$$
\begin{aligned}
g_{a p} & \simeq \frac{m_{p}}{f_{a}}\left(C_{u} \Delta u+C_{d} \Delta d-\left(\frac{m_{d}}{m_{u}+m_{d}} \Delta u+\frac{m_{u}}{m_{u}+m_{d}} \Delta d\right) c_{G}\right), \\
& \simeq \frac{m_{p}}{f_{a}}\left(0.90 C_{u}(2 \mathrm{GeV})-0.38 C_{d}(2 \mathrm{GeV})-0.48 c_{G}\right), \\
g_{a n} & \simeq \frac{m_{n}}{f_{a}}\left(C_{d} \Delta u+C_{u} \Delta d-\left(\frac{m_{u}}{m_{u}+m_{d}} \Delta u+\frac{m_{d}}{m_{u}+m_{d}} \Delta d\right) c_{G}\right), \\
& \simeq \frac{m_{n}}{f_{a}}\left(0.90 C_{d}(2 \mathrm{GeV})-0.38 C_{u}(2 \mathrm{GeV})-0.04 c_{G}\right),
\end{aligned}
$$

with $\Delta u=0.897(27), \Delta d=-0.376(27)$, and $m_{u} / m_{d}=0.48(3)$ at $\mu=2 \mathrm{GeV}$ in $\overline{\mathrm{MS}}[3,27]{ }^{4}$

\subsection{Semi-analytic solution and numerical results}

We now discuss the solution to the RG equations of the previous subsection. We are concerned with the RG corrections to the low energy couplings $C_{u}, C_{d}$ and $C_{e}$. We will provide semi-analytic formulas for them as well as showing fully numerical results.

For a small $\tan \beta(\lesssim 50)$, as a good approximation, one can keep only the top Yukawa coupling $\tilde{y}_{t}$ among the three Yukawa couplings $\tilde{y}_{t, b, \tau}$ in the RG equations (3.15). By integrating the RG equations eq. (3.15), eq. (3.24), and eq. (3.29) using this approximation, we get for a renormalization scale $\mu<m_{W}$

$$
\begin{aligned}
C_{u}(\mu) \simeq & C_{u}\left(f_{a}\right)+I_{t}\left(m_{\mathrm{BSM}}, f_{a}\right) \sin ^{2} \beta+I_{t}^{\mathrm{SM}}\left(m_{t}, m_{\mathrm{BSM}}\right) \\
& +\frac{2}{3 \pi^{2}} \int_{m_{\mathrm{BSM}}}^{f_{a}} \frac{d \mu^{\prime}}{\mu^{\prime}}\left(\alpha_{s}^{2}\left(\mu^{\prime}\right) \tilde{c}_{G}\left(\mu^{\prime}\right)+\frac{9 \sin ^{2} \beta}{16} \alpha_{2}^{2}\left(\mu^{\prime}\right) \tilde{c}_{W}\left(\mu^{\prime}\right)+\frac{\left(4+9 \sin ^{2} \beta\right)}{48} \alpha_{1}^{2}\left(\mu^{\prime}\right) \tilde{c}_{B}\left(\mu^{\prime}\right)\right), \\
& +\frac{1}{\pi^{2}} \int_{m_{W}}^{m_{\mathrm{BSM}}} \frac{d \mu^{\prime}}{\mu^{\prime}}\left(\alpha_{s}^{2}\left(\mu^{\prime}\right) \tilde{c}_{G}\left(\mu^{\prime}\right)+\frac{9}{32} \alpha_{2}^{2}\left(\mu^{\prime}\right) \tilde{c}_{W}\left(\mu^{\prime}\right)+\frac{17}{96} \alpha_{1}^{2}\left(\mu^{\prime}\right) \tilde{c}_{B}\left(\mu^{\prime}\right)\right), \\
& +\frac{1}{\pi^{2}} \int_{\mu}^{m_{W}} \frac{d \mu^{\prime}}{\mu^{\prime}}\left(\alpha_{s}^{2}\left(\mu^{\prime}\right) \tilde{c}_{G}\left(\mu^{\prime}\right)+\frac{1}{6} \alpha_{\mathrm{em}}^{2}\left(\mu^{\prime}\right) \tilde{c}_{\gamma}\left(\mu^{\prime}\right)\right),
\end{aligned}
$$

\footnotetext{
${ }^{4}$ Note that the numbers in eq. (3.34) and eq. (3.35) are a bit different from those in eq. 50 of ref. [27]. This is because here our $C_{u}$ and $C_{d}$ are not UV boundary parameters around $\mu=f_{a}$ but the renormalized parameters at $\mu=2 \mathrm{GeV}$. If we express $C_{u}(2 \mathrm{GeV})$ and $C_{d}(2 \mathrm{GeV})$ in terms of the $\mathrm{UV}$ parameters, for instance, using eq. (3.48) in the following analysis, we get better agreement with those numbers in ref. [27] except the coefficient of $C_{t}\left(f_{a}\right)$. Our coefficient of $C_{t}\left(f_{a}\right)$ from eq. (3.48) is significantly different from the one in ref. [27], because ref. [27] does not take into account the renormalization by Yukawa interactions.
} 


$$
\begin{aligned}
C_{d}(\mu) \simeq & C_{d}\left(f_{a}\right)-I_{t}\left(m_{\mathrm{BSM}}, f_{a}\right) \sin ^{2} \beta-I_{t}^{\mathrm{SM}}\left(m_{t}, m_{\mathrm{BSM}}\right) \\
& +\frac{2}{3 \pi^{2}} \int_{m_{\mathrm{BSM}}}^{f_{a}} \frac{d \mu^{\prime}}{\mu^{\prime}}\left(\alpha_{s}^{2}\left(\mu^{\prime}\right) \tilde{c}_{G}\left(\mu^{\prime}\right)+\frac{9 \cos ^{2} \beta}{16} \alpha_{2}^{2}\left(\mu^{\prime}\right) \tilde{c}_{W}\left(\mu^{\prime}\right)+\frac{\left(-2+9 \cos ^{2} \beta\right)}{48} \alpha_{1}^{2}\left(\mu^{\prime}\right) \tilde{c}_{B}\left(\mu^{\prime}\right)\right), \\
& +\frac{1}{\pi^{2}} \int_{m_{W}}^{m_{\mathrm{BSM}}} \frac{d \mu^{\prime}}{\mu^{\prime}}\left(\alpha_{s}^{2}\left(\mu^{\prime}\right) \tilde{c}_{G}\left(\mu^{\prime}\right)+\frac{9}{32} \alpha_{2}^{2}\left(\mu^{\prime}\right) \tilde{c}_{W}\left(\mu^{\prime}\right)+\frac{5}{96} \alpha_{1}^{2}\left(\mu^{\prime}\right) \tilde{c}_{B}\left(\mu^{\prime}\right)\right), \\
& +\frac{1}{\pi^{2}} \int_{\mu}^{m_{W}} \frac{d \mu^{\prime}}{\mu^{\prime}}\left(\alpha_{s}^{2}\left(\mu^{\prime}\right) \tilde{c}_{G}\left(\mu^{\prime}\right)+\frac{1}{24} \alpha_{\mathrm{em}}^{2}\left(\mu^{\prime}\right) \tilde{c}_{\gamma}\left(\mu^{\prime}\right)\right), \\
C_{e}(\mu) \simeq & C_{e}\left(f_{a}\right)-I_{t}\left(m_{\mathrm{BSM}}, f_{a}\right) \sin ^{2} \beta-I_{t}^{\mathrm{SM}}\left(m_{t}, m_{\mathrm{BSM}}\right) \\
& +\frac{2}{3 \pi^{2}} \int_{m_{\mathrm{BSM}}}^{f_{a}} \frac{d \mu^{\prime}}{\mu^{\prime}}\left(\frac{9 \cos ^{2} \beta}{16} \alpha_{2}^{2}\left(\mu^{\prime}\right) \tilde{c}_{W}\left(\mu^{\prime}\right)+\frac{3\left(2+\cos ^{2} \beta\right)}{16} \alpha_{1}^{2}\left(\mu^{\prime}\right) \tilde{c}_{B}\left(\mu^{\prime}\right)\right) \\
& +\frac{1}{\pi^{2}} \int_{m_{W}}^{m_{\mathrm{BSM}}} \frac{d \mu^{\prime}}{\mu^{\prime}}\left(\frac{9}{32} \alpha_{2}^{2}\left(\mu^{\prime}\right) \tilde{c}_{W}\left(\mu^{\prime}\right)+\frac{15}{32} \alpha_{1}^{2}\left(\mu^{\prime}\right) \tilde{c}_{B}\left(\mu^{\prime}\right)\right), \\
& +\frac{1}{\pi^{2}} \int_{\mu}^{m_{W}} \frac{d \mu^{\prime}}{\mu^{\prime}} \frac{3}{8} \alpha_{\mathrm{em}}^{2}\left(\mu^{\prime}\right) \tilde{c}_{\gamma}\left(\mu^{\prime}\right),
\end{aligned}
$$

where $C_{\Psi}\left(f_{a}\right) \equiv C_{\Psi}^{0}$ as defined in eq. $(2.5)$, and $I_{t}\left(m_{\mathrm{BSM}}, f_{a}\right)$ and $I_{t}^{\mathrm{SM}}\left(m_{t}, m_{\mathrm{BSM}}\right)$ are defined as

$$
\begin{aligned}
I_{t}\left(m_{\mathrm{BSM}}, f_{a}\right) & \equiv-\frac{3}{8 \pi^{2}} \int_{m_{\mathrm{BSM}}}^{f_{a}} \frac{d \mu^{\prime}}{\mu^{\prime}} \tilde{y}_{t}^{2}\left(\mu^{\prime}\right) n_{t}\left(\mu^{\prime}\right), \\
I_{t}^{\mathrm{SM}}\left(m_{t}, m_{\mathrm{BSM}}\right) & \equiv-\frac{3}{8 \pi^{2}} \int_{m_{t}}^{m_{\mathrm{BSM}}} \frac{d \mu^{\prime}}{\mu^{\prime}} y_{t}^{2}\left(\mu^{\prime}\right) C_{t}\left(\mu^{\prime}\right) .
\end{aligned}
$$

In the above integrations one can ignore the running of the parameters $\tilde{c}_{G}(\mu), \tilde{c}_{W}(\mu)$ and $\tilde{c}_{B}(\mu)$ as this effect is of subleading order, while one has to take into account the heavy particle threshold corrections as in eq. (3.30).

The above formulas show that the RG corrections to $C_{u, d, e}$ are mainly determined by the running parameters $n_{t}, C_{t}, \tilde{c}_{G, W, B}$. To get a more instructive form for $I_{t}$ and $I_{t}^{S M}$ from the top Yukawa interaction, one may integrate the one-loop RG equations for $\alpha_{s}(\mu)$ and the top Yukawa coupling while solving the first-order linear differential equations for $n_{t}(\mu)$ and $C_{t}(\mu)$ in eq. (3.15) and eq. (3.24). We then find

$$
\begin{aligned}
& I_{t}\left(m_{\mathrm{BSM}}, f_{a}\right) \simeq-\frac{1}{2}\left(1-R_{t}\left(m_{\mathrm{BSM}}, f_{a}\right)\right) n_{t}\left(f_{a}\right) \\
& -\frac{1}{3 \pi^{2}} \int_{m_{\mathrm{BSM}}}^{f_{a}} \frac{d \mu^{\prime}}{\mu^{\prime}}\left(1-R_{t}\left(m_{\mathrm{BSM}}, \mu^{\prime}\right)\right)\left(\alpha_{s}^{2}\left(\mu^{\prime}\right) \tilde{c}_{G}\left(\mu^{\prime}\right)+\frac{9}{16} \alpha_{2}^{2}\left(\mu^{\prime}\right) \tilde{c}_{W}\left(\mu^{\prime}\right)+\frac{13}{48} \alpha_{1}^{2}\left(\mu^{\prime}\right) \tilde{c}_{B}\left(\mu^{\prime}\right)\right), \\
& I_{t}^{\mathrm{SM}}\left(m_{t}, m_{\mathrm{BSM}}\right) \simeq-\frac{2}{3}\left(1-R_{t}^{\mathrm{SM}}\left(m_{t}, m_{\mathrm{BSM}}\right)\right) C_{t}\left(m_{\mathrm{BSM}}\right) \\
& -\frac{2}{3 \pi^{2}} \int_{m_{t}}^{m_{\mathrm{BSM}}} \frac{d \mu^{\prime}}{\mu^{\prime}}\left(1-R_{t}^{\mathrm{SM}}\left(m_{t}, \mu^{\prime}\right)\right)\left(\alpha_{s}^{2}\left(\mu^{\prime}\right) \tilde{c}_{G}\left(\mu^{\prime}\right)+\frac{9}{32} \alpha_{2}^{2}\left(\mu^{\prime}\right) \tilde{c}_{W}\left(\mu^{\prime}\right)+\frac{17}{96} \alpha_{1}^{2}\left(\mu^{\prime}\right) \tilde{c}_{B}\left(\mu^{\prime}\right)\right),
\end{aligned}
$$


with

$$
\begin{aligned}
R_{t}\left(\mu, \mu^{\prime}\right) & \equiv \exp \left[-24 \int_{\mu}^{\mu^{\prime}} \frac{d \mu^{\prime \prime}}{\mu^{\prime \prime}} \frac{\tilde{y}_{t}^{2}\left(\mu^{\prime \prime}\right)}{32 \pi^{2}}\right] \simeq\left[1-\frac{18}{7} \frac{\alpha_{t}\left(\mu^{\prime}\right)}{\alpha_{s}\left(\mu^{\prime}\right)}\left(1-\left(\frac{\alpha_{s}(\mu)}{\alpha_{s}\left(\mu^{\prime}\right)}\right)^{7 / 9}\right)\right]^{-1}, \\
R_{t}^{\mathrm{SM}}\left(\mu, \mu^{\prime}\right) & \equiv \exp \left[-18 \int_{\mu}^{\mu^{\prime}} \frac{d \mu^{\prime \prime}}{\mu^{\prime \prime}} \frac{y_{t}^{2}\left(\mu^{\prime \prime}\right)}{32 \pi^{2}}\right] \simeq\left[1-\frac{9}{2} \frac{\alpha_{t}\left(\mu^{\prime}\right)}{\alpha_{s}\left(\mu^{\prime}\right)}\left(1-\left(\frac{\alpha_{s}(\mu)}{\alpha_{s}\left(\mu^{\prime}\right)}\right)^{1 / 7}\right)\right]^{-1},
\end{aligned}
$$

where $\alpha_{t}(\mu) \equiv \tilde{y}_{t}^{2}(\mu) / 4 \pi$ for $\mu>m_{\mathrm{BSM}}$ and $\alpha_{t}(\mu) \equiv y_{t}^{2}(\mu) / 4 \pi$ for $\mu<m_{\mathrm{BSM}}$. Eq. (3.41) and eq. (3.42) explicitly show potentially important contributions from $\tilde{c}_{G, W, B}$ through the top Yukawa interaction even when $n_{t}\left(f_{a}\right)$ and $C_{t}\left(m_{\mathrm{BSM}}\right)$ vanish. As we will see below, they give rise to an important radiative correction to $C_{e}$ for KSVZ-like axions.

Eqs. (3.36), (3.37), (3.38) and (3.39) show that the radiative corrections to $C_{\Psi}$ ( $\Psi=u, d, e)$ induced by the top Yukawa interaction and the SM gauge interactions originate mainly from the four UV parameters $\left\{n_{t}\left(f_{a}\right), \tilde{c}_{G}\left(f_{a}\right), \tilde{c}_{W}\left(f_{a}\right), \tilde{c}_{B}\left(f_{a}\right)\right\}$ in the MSSM case or from $\left\{C_{t}\left(f_{a}\right), \tilde{c}_{G}\left(f_{a}\right), \tilde{c}_{W}\left(f_{a}\right), \tilde{c}_{B}\left(f_{a}\right)\right\}$ in the SM case. Then one can parameterize the low energy axion couplings to the light quarks and electron as

$$
\begin{aligned}
C_{u}(2 \mathrm{GeV}) & =C_{u}\left(f_{a}\right)+\Delta C_{u}, \\
C_{d}(2 \mathrm{GeV}) & =C_{d}\left(f_{a}\right)+\Delta C_{d}, \\
C_{e}\left(m_{e}\right) & =C_{e}\left(f_{a}\right)+\Delta C_{e},
\end{aligned}
$$

where $C_{\Psi}\left(f_{a}\right)(\Psi=u, d, e)$ are identified as the tree-level values $C_{\Psi}^{0}$ and

$$
\begin{aligned}
\mathrm{SM}: & \Delta C_{\Psi}=r_{\Psi}^{t} C_{t}\left(f_{a}\right)+r_{\Psi}^{G} \tilde{c}_{G}\left(f_{a}\right)+r_{\Psi}^{W} \tilde{c}_{W}\left(f_{a}\right)+r_{\Psi}^{B} \tilde{c}_{B}\left(f_{a}\right), \\
\mathrm{MSSM}: & \Delta C_{\Psi}=r_{\Psi}^{t} n_{t}\left(f_{a}\right)+r_{\Psi}^{G} \tilde{c}_{G}\left(f_{a}\right)+r_{\Psi}^{W} \tilde{c}_{W}\left(f_{a}\right)+r_{\Psi}^{B} \tilde{c}_{B}\left(f_{a}\right) .
\end{aligned}
$$

To compute the coefficients $r_{\Psi}^{X}(X=t, G, W, B)$ in this parameterization, we solve the RG equations in a fully numerical way with the running SM gauge couplings and top Yukawa coupling at two-loop order. The resulting $r_{\Psi}^{X}$ are depicted in figure 4 for the SM case (left panel) and the MSSM case (right panel). For the MSSM, we choose $\tan \beta=10$ and the SUSY particle masses $m_{\mathrm{SUSY}}=10 \mathrm{TeV}$. Our results show that

$$
\begin{aligned}
& r_{u, d, e}^{t} \sim \text { few } \times 10^{-1}, \quad \quad r_{u, d}^{G} \sim 10^{-2}, \quad r_{e}^{G} \sim \text { few } \times 10^{-4}-10^{-3}, \\
& r_{u, d, e}^{W} \sim \text { few } \times 10^{-4}-10^{-3}, \quad r_{u, d}^{B} \sim \text { few } \times 10^{-5}-10^{-4}, \quad r_{e}^{B} \sim 10^{-4}
\end{aligned}
$$

for $10^{7} \mathrm{GeV} \lesssim f_{a} \lesssim 10^{16} \mathrm{GeV}$. For example, for $f_{a}=10^{10} \mathrm{GeV}$ in the SM case, we find

$$
\begin{aligned}
C_{u}(2 \mathrm{GeV}) & \simeq C_{u}\left(f_{a}\right)-0.28 C_{t}\left(f_{a}\right)+\left[17.8 \tilde{c}_{G}\left(f_{a}\right)+0.33 \tilde{c}_{W}\left(f_{a}\right)+0.032 \tilde{c}_{B}\left(f_{a}\right)\right] \times 10^{-3}, \\
C_{d}(2 \mathrm{GeV}) & \simeq C_{d}\left(f_{a}\right)+0.30 C_{t}\left(f_{a}\right)+\left[19.5 \tilde{c}_{G}\left(f_{a}\right)+0.48 \tilde{c}_{W}\left(f_{a}\right)+0.017 \tilde{c}_{B}\left(f_{a}\right)\right] \times 10^{-3}, \\
C_{e}\left(m_{e}\right) & \simeq C_{e}\left(f_{a}\right)+0.29 C_{t}\left(f_{a}\right)+\left[0.80 \tilde{c}_{G}\left(f_{a}\right)+0.54 \tilde{c}_{W}\left(f_{a}\right)+0.13 \tilde{c}_{B}\left(f_{a}\right)\right] \times 10^{-3},
\end{aligned}
$$

and for $f_{a}=10^{10} \mathrm{GeV}, m_{\mathrm{SUSY}}=10 \mathrm{TeV}$ and $\tan \beta=10$ in the MSSM case,

$$
\begin{aligned}
C_{u}(2 \mathrm{GeV}) & \simeq C_{u}\left(f_{a}\right)-0.28 n_{t}\left(f_{a}\right)+\left[17.7 \tilde{c}_{G}\left(f_{a}\right)+0.52 \tilde{c}_{W}\left(f_{a}\right)+0.036 \tilde{c}_{B}\left(f_{a}\right)\right] \times 10^{-3}, \\
C_{d}(2 \mathrm{GeV}) & \simeq C_{d}\left(f_{a}\right)+0.31 n_{t}\left(f_{a}\right)+\left[19.4 \tilde{c}_{G}\left(f_{a}\right)+0.23 \tilde{c}_{W}\left(f_{a}\right)+0.0047 \tilde{c}_{B}\left(f_{a}\right)\right] \times 10^{-3}, \\
C_{e}\left(m_{e}\right) & \simeq C_{e}\left(f_{a}\right)+0.29 n_{t}\left(f_{a}\right)+\left[0.81 \tilde{c}_{G}\left(f_{a}\right)+0.28 \tilde{c}_{W}\left(f_{a}\right)+0.10 \tilde{c}_{B}\left(f_{a}\right)\right] \times 10^{-3} .
\end{aligned}
$$



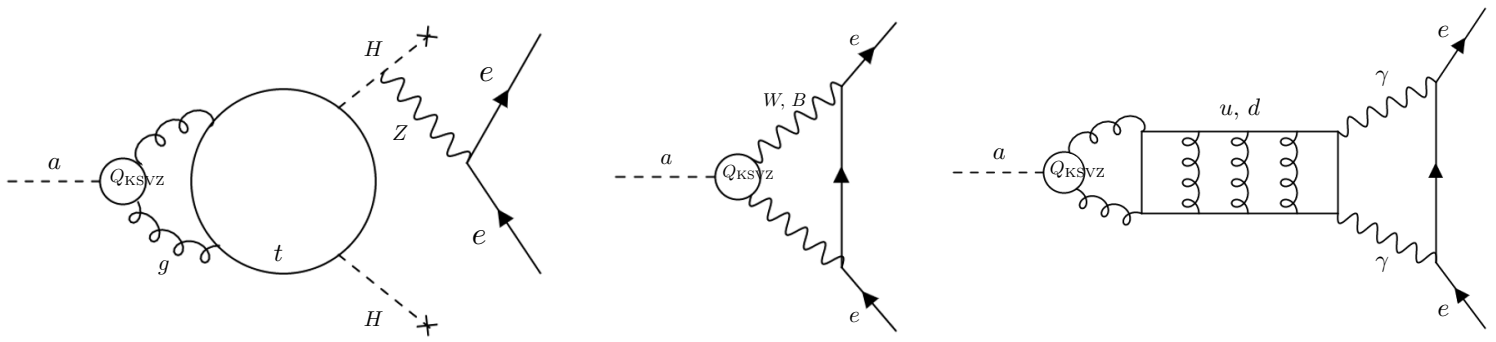

Figure 3. Diagrams for the radiative corrections to the axion-electron coupling $C_{e}$ in KSVZlike models.

Here the difference in the value of $r_{\Psi}^{W}$ between the SM case and the MSSM case is mainly due to a different running of the gauge couplings and top Yukawa coupling.

A particularly interesting aspect of our results is that the axion-electron coupling $C_{e}$ receives a large radiative correction from the UV parameter $\tilde{c}_{G}\left(f_{a}\right)$, which was first shown in [25] for the SM case to our knowledge. This has an important implication for KSVZ-like QCD axions. For KSVZ-like axions, $n_{t}\left(f_{a}\right)=C_{t}\left(f_{a}\right)=0$ so that there is no one-loop level radiative correction from the Yukawa interactions. In the previous literature, the leading contribution to $C_{e}$ in KSVZ axion model was thought to be from non-zero $c_{W}$ or $c_{B}$ as in the second diagram of figure 3 , or from the axion-pion mixing below the QCD scale as in the third diagram of figure 3 for a minimal KSVZ model with $c_{W}=c_{B}=0$ [19,20]. But the results eq. (3.48) and eq. (3.49) indicate that there is a larger contribution from the axiongluon coupling $c_{G}$. This radiative correction is originated from the first diagram of figure 3 involving the exotic heavy quark $\left(\mathcal{Q}, \mathcal{Q}^{c}\right)$, gluons, top quark and Higgs doublet at threeloop level. This process is encoded in $I_{t}$ and $I_{t}^{\mathrm{SM}}$ (eq. (3.41) and eq. (3.42)) of eq. (3.38). Although it is a three-loop process, it beats the contributions from the second and third diagrams in figure 3 because $\alpha_{s}^{2} \gg \alpha_{2,1}^{2}$ and $y_{t} \sim \mathcal{O}(1)$. For instance, for KSVZ-like axions with $m_{\mathcal{Q}}=10^{10} \mathrm{GeV}$, we find

$$
\begin{aligned}
& C_{e}\left(m_{e}\right) \simeq\left[0.84 c_{G}-0.03 c_{G}+0.28 c_{W}+0.10 c_{B}\right] \times 10^{-3}(\mathrm{KSVZ} \text { with MSSM }) \\
& C_{e}\left(m_{e}\right) \simeq\left[0.83 c_{G}-0.03 c_{G}+0.54 c_{W}+0.13 c_{B}\right] \times 10^{-3}(\mathrm{KSVZ} \text { with SM })
\end{aligned}
$$

where the first term in the square bracket denotes the contribution from the first diagram in figure 3, the second is the contribution from the axion-pion mixing represented by the last diagram in figure 3 , and the last two terms are the contributions from the two-loop diagrams involving the electroweak gauge bosons.

\section{Distinguishing the axions by low energy precision physics}

Based on the results of the previous sections, we are going to discuss the possibility to distinguish the different classes of axion models experimentally. As discussed in section 2, one can consider three classes of axion models: KSVZ-like, DFSZ-like, and string-theoretic axions. They may show different patterns of the low energy axion couplings to the nucleons and electron after properly taking into account the relevant radiative corrections. To 

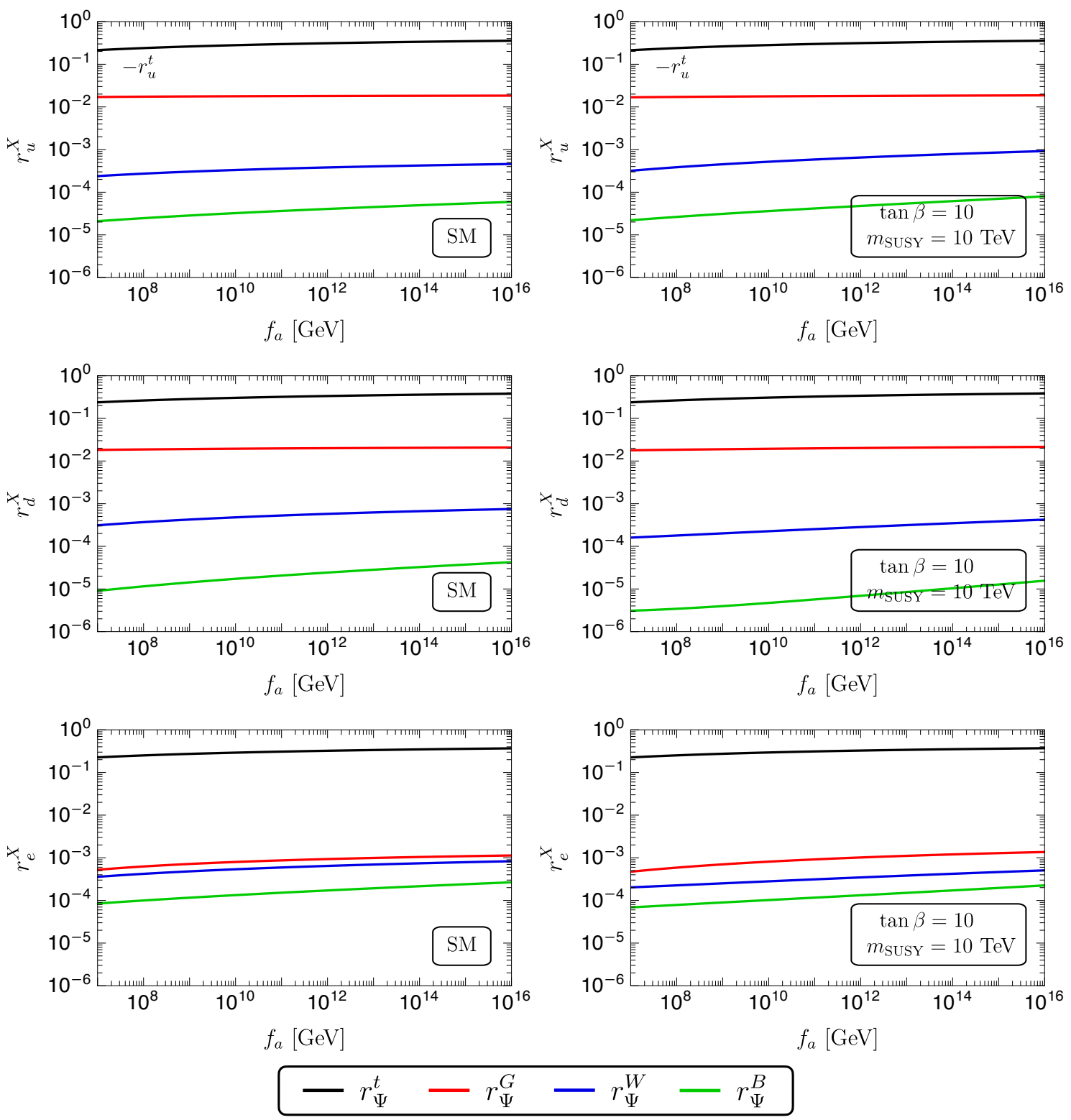

Figure 4. Radiative corrections to the axion couplings to the light quarks and electron from $\left\{C_{t}\left(f_{a}\right), \tilde{c}_{G}\left(f_{a}\right), \tilde{c}_{W}\left(f_{a}\right), \tilde{c}_{B}\left(f_{a}\right)\right\}$ for the SM or from $\left\{n_{t}\left(f_{a}\right), \tilde{c}_{G}\left(f_{a}\right), \tilde{c}_{W}\left(f_{a}\right), \tilde{c}_{B}\left(f_{a}\right)\right\}$ for the MSSM: $\Delta C_{\Psi}=r_{\Psi}^{t}\left[n_{t}\left(f_{a}\right)\right.$ or $\left.C_{t}\left(f_{a}\right)\right]+\sum_{A=G, W, B} r_{\Psi}^{A} \tilde{c}_{A}\left(f_{a}\right)$. For the MSSM, we take the parameters $\tan \beta=10$ and $m_{\mathrm{SUSY}}=10 \mathrm{TeV}$. 
include string-theoretic axions in a consistent manner, in the following we discuss the coupling patterns in the MSSM framework which assumes $N=1$ SUSY at scales around $f_{a}$.

Let us first consider the approximate expression of the low energy axion couplings defined in eq. (3.31) for a specific parameter point with $f_{a}=10^{10} \mathrm{GeV}, m_{\mathrm{SUSY}}=10 \mathrm{TeV}$ and $\tan \beta=10$. Applying the numerical result in eq. (3.49) to eq. (3.34) and eq. (3.35), we find

$$
\begin{aligned}
g_{a p} \simeq \frac{m_{p}}{f_{a}}[ & -0.48 c_{G}+0.90 C_{u}^{0}-0.38 C_{d}^{0} \\
& \left.\quad-0.37 n_{t}\left(f_{a}\right)+\left(8.6 \tilde{c}_{G}\left(f_{a}\right)+0.38 \tilde{c}_{W}\left(f_{a}\right)+0.031 \tilde{c}_{B}\left(f_{a}\right)\right) \times 10^{-3}\right], \\
g_{a n} \simeq \frac{m_{n}}{f_{a}}[ & -0.037 c_{G}-0.38 C_{u}^{0}+0.90 C_{d}^{0} \\
& \left.+0.38 n_{t}\left(f_{a}\right)+\left(11 \tilde{c}_{G}\left(f_{a}\right)+0.0078 \tilde{c}_{W}\left(f_{a}\right)-0.0094 \tilde{c}_{B}\left(f_{a}\right)\right) \times 10^{-3}\right], \\
g_{a e} \simeq \frac{m_{e}}{f_{a}}[ & \left.C_{e}^{0}+0.29 n_{t}\left(f_{a}\right)+\left(0.81 \tilde{c}_{G}\left(f_{a}\right)+0.28 \tilde{c}_{W}\left(f_{a}\right)+0.10 \tilde{c}_{B}\left(f_{a}\right)\right) \times 10^{-3}\right],
\end{aligned}
$$

where $C_{u}^{0}, C_{d}^{0}$ and $C_{e}^{0}$ are the tree level couplings as defined in eq. (2.5), and the contributions from $n_{t}\left(f_{a}\right)$ and $\tilde{c}_{A}\left(f_{a}\right)(A=G, W, B)$ represent the radiative corrections. On the other hand, the axion-photon coupling $g_{a \gamma}$ at low energies below the QCD scale is given by eq. (3.32),

$$
g_{a \gamma} \simeq \frac{\alpha_{\mathrm{em}}}{2 \pi} \frac{1}{f_{a}}\left(c_{W}+c_{B}-1.92 c_{G}\right)
$$

As we have defined in section 2, we refer to DFSZ-like axions as generic field-theoretic axions with $C_{\Psi}^{0}=\mathcal{O}(1)(\Psi=u, d, e)$. Thus for SUSY DFSZ-like axions with $f_{a}=10^{10} \mathrm{GeV}$, $m_{\mathrm{SUSY}}=10 \mathrm{TeV}$ and $\tan \beta=10$,

$$
\begin{aligned}
g_{a p}^{\mathrm{DFSZ}} & \simeq \frac{m_{p}}{f_{a}}\left[-0.48 c_{G}+0.90 C_{u}^{0}-0.38 C_{d}^{0}-0.37 n_{t}\left(f_{a}\right)\right] \sim \frac{m_{p}}{f_{a}} \mathcal{O}(1), \\
g_{a n}^{\mathrm{DFSZ}} & \simeq \frac{m_{n}}{f_{a}}\left[-0.037 c_{G}-0.38 C_{u}^{0}+0.90 C_{d}^{0}+0.38 n_{t}\left(f_{a}\right)\right] \sim \frac{m_{n}}{f_{a}} \mathcal{O}(1), \\
g_{a e}^{\mathrm{DFSZ}} & \simeq \frac{m_{e}}{f_{a}}\left[C_{e}^{0}+0.29 n_{t}\left(f_{a}\right)\right] \sim \frac{m_{e}}{f_{a}} \mathcal{O}(1) .
\end{aligned}
$$

Here we note $n_{t}\left(f_{a}\right)=0$ for the standard DFSZ models of eq. (2.13) and eq. (2.15), while it can be non-vanishing for more generic DFSZ-like axions [34]. In fact, for the standard DFSZ axions, $n_{t}\left(f_{a}\right)=\tilde{c}_{A}\left(f_{a}\right)=0(A=G, W, B)$, and the RG running is present only below the BSM scale after the BSM Higgs doublet or Higgsinos are integrated out giving rise to a non-zero $C_{t}\left(m_{\mathrm{BSM}}\right)$ or $\tilde{c}_{W, B}\left(m_{\mathrm{BSM}}\right)$, while the running by $\tilde{c}_{G}$ is present only after the top quark is integrated out. Thus the RG running effect is mostly negligible for the standard DFSZ models, while there could be a sizable RG correction from the top Yukawa coupling for more generic DFSZ-like axions having a non-zero $n_{t}\left(f_{a}\right)$.

On the other hand, KSVZ-like axions are defined as generic field-theoretic axions having vanishing tree level couplings to the SM particles and Higgs doublets, i.e. $C_{u}^{0}=$ $C_{d}^{0}=n_{t}\left(f_{a}\right)=0$ (or more generically $\lesssim \mathcal{O}\left(\left(g_{\text {GUT }}^{2} / 8 \pi^{2}\right)^{2}\right)$ and $\tilde{c}_{A}\left(f_{a}\right)=c_{A}(A=G, W, B)$. 
Thus for SUSY KSVZ-like axions with $f_{a}=10^{10} \mathrm{GeV}, m_{\mathrm{SUSY}}=10 \mathrm{TeV}$ and $\tan \beta=10$,

$$
\begin{aligned}
& g_{a p}^{\mathrm{KSVZ}} \simeq \frac{m_{p}}{f_{a}}\left[-0.48 c_{G}+\left(0.38 c_{W}+0.031 c_{B}\right) \times 10^{-3}\right], \\
& g_{a n}^{\mathrm{KSVZ}} \simeq \frac{m_{n}}{f_{a}}\left[-0.026 c_{G}+\left(0.78 c_{W}-0.94 c_{B}\right) \times 10^{-5}\right], \\
& g_{a e}^{\mathrm{KSVZ}} \simeq \frac{m_{e}}{f_{a}}\left(0.81 c_{G}+0.28 c_{W}+0.10 c_{B}\right) \times 10^{-3} .
\end{aligned}
$$

In fact here it is assumed that the mass of the exotic heavy quark $\mathcal{Q}+\mathcal{Q}^{c}$ in the model (2.16), i.e. $m_{\mathcal{Q}}=y_{\mathcal{Q}} f_{a} / \sqrt{2}$, is around $f_{a}$. If $m_{\mathcal{Q}} \ll f_{a}$ with $y_{\mathcal{Q}} \ll 1, \tilde{c}_{A}\left(f_{a}\right)=c_{A}-c_{\mathcal{Q}}-c_{\mathcal{Q}^{c}}=0$, and the RG running of axion couplings starts from the scale $\mu=m_{\mathcal{Q}}\left(\ll f_{a}\right)$ at which $\mathcal{Q}+\mathcal{Q}^{c}$ is integrated out, yielding $\tilde{c}_{A}\left(m_{\mathcal{Q}}\right)=c_{A}$. For this case, the coefficients in eq. (4.4) depends on $m_{\mathcal{Q}}$ rather than $f_{a}$. In the following analysis, we will also consider the case $m_{\mathcal{Q}} \ll f_{a}$.

String-theoretic axions have a distinctive feature that their couplings to matter fields are suppressed by $1 / \tau$ where $\tau$ denotes the moduli partner whose vacuum expectation value can be identified as the Euclidean action of the brane instanton coupled to string-theoretic axion. As a consequence, $C_{\Psi}^{0}(\Psi=u, d, e)$ are numerically of $\mathcal{O}\left(g_{\text {GUT }}^{2} / 8 \pi^{2}\right)$ for light stringtheoretic axions which couple to the gluons or the photon, including a QCD axion and an ultralight ALP coupled to the photon (See eqs. (2.34) and (2.35)). As discussed in section 2, one can parameterize the string-theoretic axion couplings to matter fields at $\mu \sim f_{a}$ as

$$
c_{\phi}\left(f_{a}\right)=\omega_{\phi} \frac{g_{\mathrm{GUT}}^{2}}{16 \pi^{2}}, \quad c_{\psi}=\omega_{\psi} \frac{g_{\mathrm{GUT}}^{2}}{16 \pi^{2}},
$$

where the model-dependent coefficients $\omega_{\phi}$ and $\omega_{\psi}$ are expected to be of order unity. We then find for string-theoretic axions with $f_{a}=10^{10} \mathrm{GeV}, m_{\mathrm{SUSY}}=10 \mathrm{TeV}$ and $\tan \beta=10$,

$$
\begin{aligned}
& g_{a p}^{\text {string }} \simeq \frac{m_{p}}{f_{a}}\left[g_{\text {GUT }}^{2}\left(0.56\left(\omega_{u_{1}^{c}}+\omega_{H_{u}}\right)+0.32 \omega_{Q_{1}}-0.24\left(\omega_{Q_{3}}+\omega_{u_{3}^{c}}+\omega_{d_{1}^{c}}\right)\right) \times 10^{-2}\right. \\
& \left.-0.48 c_{G}+\left(0.38 c_{W}+0.031 c_{B}\right) \times 10^{-3}\right], \\
& g_{a n}^{\text {string }} \simeq \frac{m_{n}}{f_{a}}\left[g_{\mathrm{GUT}}^{2}\left(0.56\left(\omega_{d_{1}^{c}}-\omega_{H_{u}}\right)+0.32 \omega_{Q_{1}}+0.24\left(\omega_{Q_{3}}+\omega_{u_{3}^{c}}-\omega_{u_{1}^{c}}\right)\right) \times 10^{-2}\right. \\
& \left.-0.026 c_{G}+\left(0.78 c_{W}-0.94 c_{B}\right) \times 10^{-5}\right], \\
& g_{a e}^{\text {string }} \simeq \frac{m_{e}}{f_{a}}\left[g_{\mathrm{GUT}}^{2}\left(0.63\left(\omega_{e_{1}^{c}}+\omega_{L_{1}}\right)-0.44 \omega_{H_{u}}+0.19\left(\omega_{Q_{3}}+\omega_{u_{3}^{c}}\right)\right) \times 10^{-2}\right. \\
& \left.+\left(0.81 c_{G}+0.28 c_{W}+0.10 c_{B}\right) \times 10^{-3}\right] .
\end{aligned}
$$

From the above results, one can see that the couplings show qualitatively different patterns depending on whether $c_{G} \neq 0$ (as for the QCD axion) or $c_{G}=0$. Therefore let us consider the two cases separately.

For the case with $c_{G} \neq 0$ that includes the QCD axion, all of the three classes of axions have a similar value of the coupling ratio $g_{a p} / g_{a \gamma}$. On the other hand, the coupling ratio $g_{a n} / g_{a \gamma}$ for KSVZ-like QCD axions and string-theoretic QCD axions is an order of magnitude smaller than that of DFSZ-like QCD axions. So one can discriminate between those two classes of models with an experimental measurement of $g_{a n} / g_{a \gamma}$. To completely 

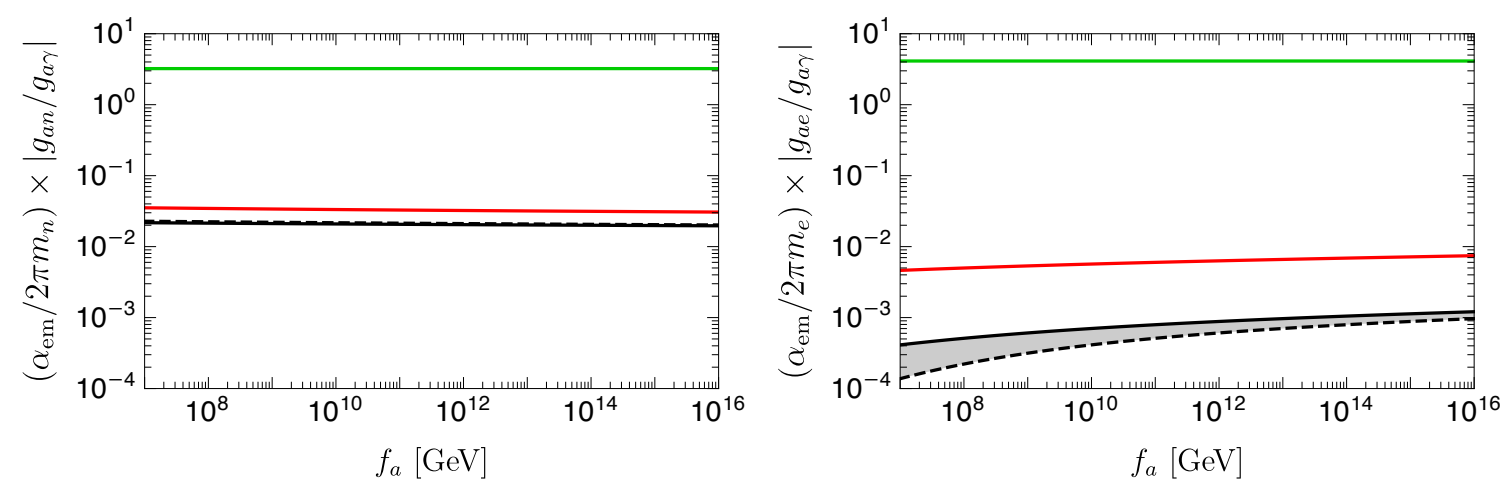

Figure 5. The predicted ratios of the axion couplings from the minimal QCD axion models within the MSSM framework (green: DFSZ-like axions, black: KSVZ-like axions, red: stringtheoretic axions). For KSVZ-like axions, the heavy exotic quark is assumed to have a mass between $10^{-3} f_{a}$ (dashed black) and $f_{a}$ (solid black). For the MSSM parameters, we take $\tan \beta=10$ and $m_{\mathrm{SUSY}}=10 \mathrm{TeV}$.

distinguish among the three classes of QCD axions, one needs to measure another coupling ratio $g_{a e} / g_{a \gamma}$ as it is predicted to be of different orders of magnitude depending on the classes of axions. In figure 5, we numerically plot the predicted coupling ratios $g_{a n} / g_{a \gamma}$ and $g_{a e} / g_{a \gamma}$ for each class of QCD axions over $f_{a}$ between $10^{7} \mathrm{GeV}$ and $10^{16} \mathrm{GeV}$. For DFSZ-like QCD axion, we use the model specified in eq. (2.14) and eq. (2.15) as a benchmark model. For KSVZ-like QCD axion, we use the model in eq. (2.16) with an $\mathrm{SU}(2)_{L^{-}}$-singlet exotic heavy quark $\mathcal{Q}$ carrying a $\mathrm{U}(1)_{Y}$ hypercharge $Y(\mathcal{Q})=-1 / 3$, which implies $c_{G}=1, c_{W}=0, c_{B}=2 / 3$. Moreover, we assume the exotic heavy quark has a mass $m_{\mathcal{Q}}$ between $10^{-3} f_{a}$ and $f_{a}$, which corresponds to the scale at which the axion coupling starts to run for this class of models. For a benchmark model of string-theoretic QCD axions, we use the large volume scenario model in eq. (2.51) with a universal scaling weight $\omega_{I}=1 / 2$ as in eq. (2.57), and also $g_{\text {GUT }}^{2} \simeq 1 / 2$ with the GUT relation $c_{G}=c_{W}=(3 / 5) c_{B}$.

On the other hand, for axions with $c_{G}=0$, but $c_{W} \neq 0$ and/or $c_{B} \neq 0$, e.g. an ALP coupled to the photon without a coupling to the gluons, eqs. (4.3)-(4.6) tell us that all the three low energy axion couplings may distinguish among the different classes of ALP models. In figure 6 , we numerically plot the predicted coupling ratios $g_{a Y} / g_{a \gamma}(Y=p, n, e)$ for each class of ALP. As an illustrative example, we use the same parameter values as in figure 5 but with different $c_{A}(A=G, W, B)$, i.e. $c_{G}=c_{B}=0$ and $c_{W}=1$ for the left panel, while $c_{G}=c_{W}=0$ and $c_{B}=1$ for the right panel. For DFSZ-like ALP, a specific model for such parameter values may include additional heavy exotic quarks that cancel the gluon anomaly of the model in eq. (2.14) and eq. (2.15). For KSVZ-like ALP, we use a similar model as in eq. (2.16) with a heavy color-singlet exotic fermion charged under $\mathrm{SU}(2)_{L}$ only (for the left plot) or $\mathrm{U}(1)_{Y}$ only (for the right plot), which is assumed to have a mass between $10^{-3} f_{a}$ and $f_{a}$. Figure 6 shows that the axion-proton coupling may be yet hard to discriminate string-theoretic ALPs from KSVZ-like ALPs for $c_{W} \neq 0$ since the radiative correction becomes sizable if $f_{a}$ is near the GUT scale. 

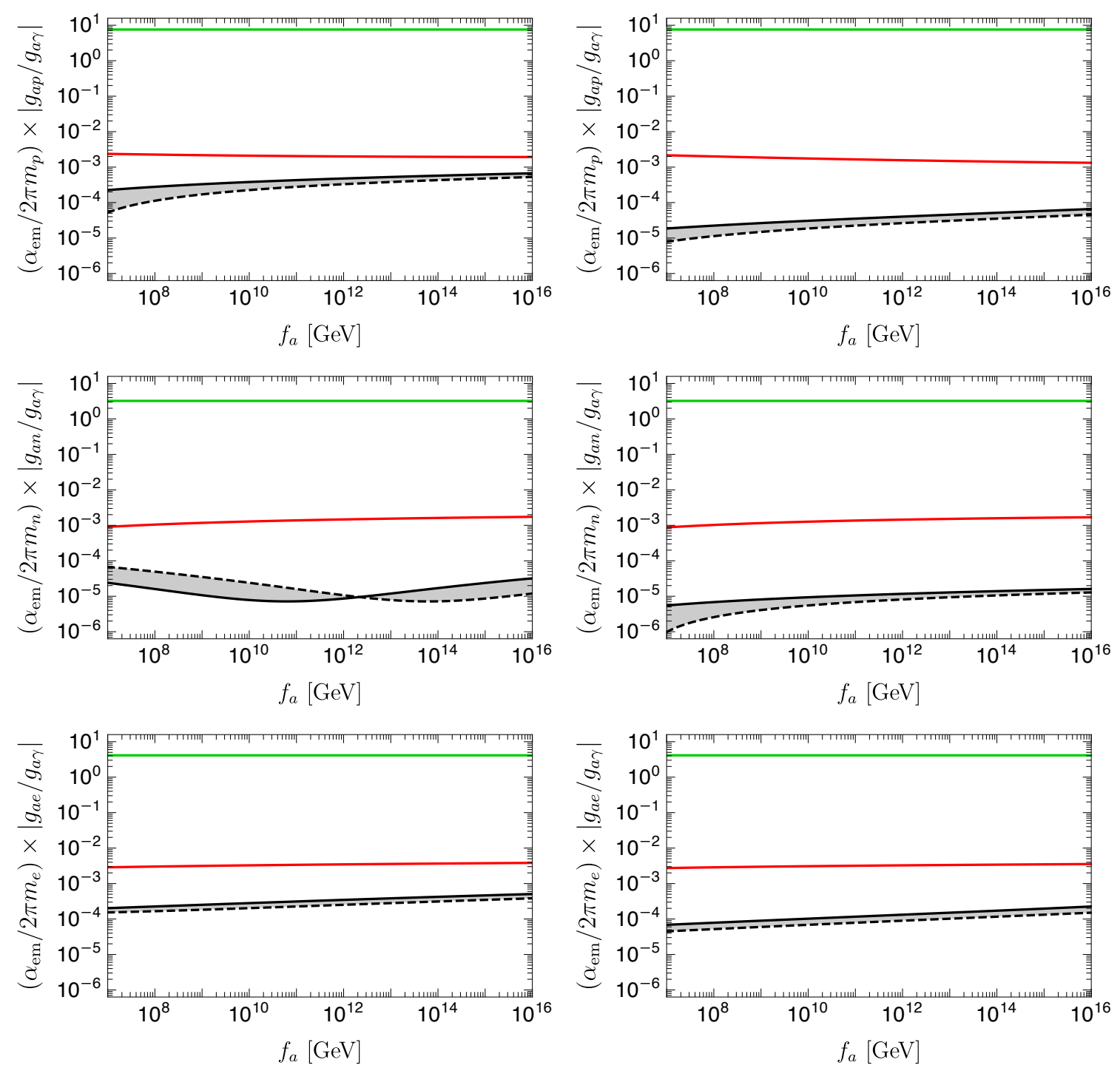

Figure 6. The predicted ratios of the ALP couplings from the minimal axion models with $c_{W}=1\left(c_{G}=c_{B}=0\right)$ (left) and $c_{B}=1\left(c_{G}=c_{W}=0\right)$ (right) within the MSSM framework (green: DFSZ-like axions, black: KSVZ-like axions, red: string-theoretic axions). For KSVZ-like axions, the heavy color-singlet exotic fermion charged under $\mathrm{SU}(2)_{L}$ (left) or $\mathrm{U}(1)_{Y}$ (right) is assumed to have a mass between $10^{-3} f_{a}$ (dashed black) and $f_{a}$ (solid black). For the MSSM parameters, we take $\tan \beta=10$ and $m_{\mathrm{SUSY}}=10 \mathrm{TeV}$.

\section{Conclusions}

Axions are compact scalar fields postulated to solve various issues in particle physics and cosmology including the strong $\mathrm{CP}$ problem and dark matter. Axions may originate from the phase of complex scalar fields (field-theoretic axions) or from the zero modes of an antisymmetric tensor ( $p$-form) gauge field (string-theoretic axions) which couples to a $(p-1)$-brane in the underlying UV theory. Axion couplings which are most relevant for an experimental detection of axions are those to the photon, nucleons and electron at scale 
well below the QCD scale, i.e. $g_{a X}(X=\gamma, p, n, e)$ which are determined mostly by the quantized couplings to the photon and gluons, as well as the couplings to the light quarks and electron around the QCD scale. Then, depending on the UV origin of the field variable and the pattern of low energy couplings, axions can be categorized into three classes: i) field-theoretic DFSZ-like axions with $R_{0}=\mathcal{O}(1)$, ii) field-theoretic KSVZ-like axions with $R_{0} \lesssim \mathcal{O}\left(\left(g_{\text {GUT }}^{2} / 16 \pi^{2}\right)^{2}\right)$, and iii) string-theoretic axions with $R_{0}=\mathcal{O}\left(g_{\text {GUT }}^{2} / 16 \pi^{2}\right)$, where $g_{\text {GUT }}$ is the gauge coupling at scales around the axion decay constant $f_{a}$, and $R_{0}$ denotes the ratio (in an appropriate unit) between the coupling to the photon (or gluons) and the tree-level couplings to the light quarks and electron. Compared to the other two, DFSZ-like axions have a clearly distinguishable pattern of $g_{a X}(X=\gamma, p, n, e)$. However it requires a careful analysis of radiative corrections to the axion couplings to the light quarks and electron to discriminate string-theoretic axions from KSVZ-like axions by experimental measurements of $g_{a X}$ or their ratios.

With this motivation, we first performed a generic renormalization group analysis for the axion couplings to matter fermions and Higgs fields while taking into account that the Standard Model can be extended to its supersymmetric extension at a scale below $f_{a}$. We then applied our results to string-theoretic axions and KSVZ-like axions to examine if they can have a distinguishable pattern of $g_{a X}(X=\gamma, p, n, e)$. We find that discriminating string-theoretic QCD axion from KSVZ-like QCD axion appears to be quite challenging, but yet it looks feasible with a significantly different value of the coupling ratio $g_{a e} / g_{a \gamma}$. For an axion-like particle (ALP) which does not couple to the gluons, but has a non-zero coupling to the photon, the prospect for discrimination is much more promising: all of the three couplings ratios $g_{a Y} / g_{a \gamma}(Y=p, n, e)$ for string-theoretic ALP and KSVZ-like ALP differ by about one order of magnitude. We also find that the coupling of KSVZ-like QCD axion to the electron is dominated by a three-loop contribution involving the heavy exotic quark, gluons, top quarks and Higgs doublet, which was not noticed in the previous studies.

\section{Acknowledgments}

This work was supported by IBS under the project code, IBS-R018-D1.

Open Access. This article is distributed under the terms of the Creative Commons Attribution License (CC-BY 4.0), which permits any use, distribution and reproduction in any medium, provided the original author(s) and source are credited.

\section{References}

[1] J.E. Kim and G. Carosi, Axions and the Strong CP Problem, Rev. Mod. Phys. 82 (2010) 557 [Erratum ibid. 91 (2019) 049902] [arXiv:0807.3125] [INSPIRE].

[2] D.J.E. Marsh, Axion Cosmology, Phys. Rept. 643 (2016) 1 [arXiv:1510.07633] [InSPIRE].

[3] L. Di Luzio, M. Giannotti, E. Nardi and L. Visinelli, The landscape of QCD axion models, Phys. Rept. 870 (2020) 1 [arXiv: 2003.01100] [InSPIRE].

[4] R.D. Peccei and H.R. Quinn, CP Conservation in the Presence of Instantons, Phys. Rev. Lett. 38 (1977) 1440 [INSPIRE]. 
[5] S. Weinberg, A New Light Boson?, Phys. Rev. Lett. 40 (1978) 223 [InSPIRE].

[6] F. Wilczek, Problem of Strong P and T Invariance in the Presence of Instantons, Phys. Rev. Lett. 40 (1978) 279 [INSPIRE].

[7] J. Preskill, M.B. Wise and F. Wilczek, Cosmology of the Invisible Axion, Phys. Lett. B 120 (1983) 127 [INSPIRE].

[8] L.F. Abbott and P. Sikivie, A Cosmological Bound on the Invisible Axion, Phys. Lett. B 120 (1983) 133 [INSPIRE].

[9] M. Dine and W. Fischler, The Not So Harmless Axion, Phys. Lett. B 120 (1983) 137 [INSPIRE].

[10] L. Hui, Wave Dark Matter, arXiv:2101.11735 [INSPIRE].

[11] J.E. Kim, Weak Interaction Singlet and Strong CP Invariance, Phys. Rev. Lett. 43 (1979) 103 [INSPIRE].

[12] M.A. Shifman, A.I. Vainshtein and V.I. Zakharov, Can Confinement Ensure Natural CP Invariance of Strong Interactions?, Nucl. Phys. B 166 (1980) 493 [INSPIRE].

[13] M. Dine, W. Fischler and M. Srednicki, A Simple Solution to the Strong CP Problem with a Harmless Axion, Phys. Lett. B 104 (1981) 199 [INSPIRE].

[14] A.R. Zhitnitsky, On Possible Suppression of the Axion Hadron Interactions (in Russian), Sov. J. Nucl. Phys. 31 (1980) 260 [inSPIRE].

[15] K. Choi, S.H. Im and C.S. Shin, Recent progress in physics of axions or axion-like particles, arXiv: 2012.05029 [INSPIRE].

[16] P. Svrček and E. Witten, Axions In String Theory, JHEP 06 (2006) 051 [hep-th/0605206] [INSPIRE].

[17] A. Arvanitaki, S. Dimopoulos, S. Dubovsky, N. Kaloper and J. March-Russell, String Axiverse, Phys. Rev. D 81 (2010) 123530 [arXiv:0905.4720] [INSPIRE].

[18] E. Palti, The Swampland: Introduction and Review, Fortsch. Phys. 67 (2019) 1900037 [arXiv: 1903.06239] [INSPIRE].

[19] M. Srednicki, Axion Couplings to Matter. 1. CP Conserving Parts, Nucl. Phys. B 260 (1985) 689 [INSPIRE].

[20] S. Chang and K. Choi, Hadronic axion window and the big bang nucleosynthesis, Phys. Lett. B 316 (1993) 51 [hep-ph/9306216] [INSPIRE].

[21] K. Choi, S.H. Im, C.B. Park and S. Yun, Minimal Flavor Violation with Axion-like Particles, JHEP 11 (2017) 070 [arXiv:1708.00021] [INSPIRE].

[22] J. Martin Camalich, M. Pospelov, P.N.H. Vuong, R. Ziegler and J. Zupan, Quark Flavor Phenomenology of the QCD Axion, Phys. Rev. D 102 (2020) 015023 [arXiv:2002.04623] [INSPIRE].

[23] M. Heiles, M. König and M. Neubert, Effective Field Theory for Heavy Vector Resonances Coupled to the Standard Model, arXiv:2011.08205 [INSPIRE].

[24] M. Chala, G. Guedes, M. Ramos and J. Santiago, Running in the ALPs, Eur. Phys. J. C 81 (2021) 181 [arXiv: 2012.09017] [INSPIRE].

[25] M. Bauer, M. Neubert, S. Renner, M. Schnubel and A. Thamm, The Low-Energy Effective Theory of Axions and ALPs, JHEP 04 (2021) 063 [arXiv: 2012.12272] [INSPIRE]. 
[26] H. Georgi, D.B. Kaplan and L. Randall, Manifesting the Invisible Axion at Low-energies, Phys. Lett. B 169 (1986) 73 [InSPIRE].

[27] G. Grilli di Cortona, E. Hardy, J. Pardo Vega and G. Villadoro, The QCD axion, precisely, JHEP 01 (2016) 034 [arXiv: 1511.02867] [INSPIRE].

[28] J.E. Kim and H.P. Nilles, The $\mu$-problem and the strong CP-problem, Phys. Lett. B 138 (1984) 150 [INSPIRE].

[29] N. Arkani-Hamed, L. Motl, A. Nicolis and C. Vafa, The String landscape, black holes and gravity as the weakest force, JHEP 06 (2007) 060 [hep-th/0601001] [INSPIRE].

[30] M. Giannotti, I.G. Irastorza, J. Redondo, A. Ringwald and K. Saikawa, Stellar Recipes for Axion Hunters, JCAP 10 (2017) 010 [arXiv:1708.02111] [INSPIRE].

[31] G. Ballesteros, J. Redondo, A. Ringwald and C. Tamarit, Standard Model-axion-seesaw-Higgs portal inflation. Five problems of particle physics and cosmology solved in one stroke, JCAP 08 (2017) 001 [arXiv:1610.01639] [INSPIRE].

[32] L. Di Luzio, F. Mescia and E. Nardi, Redefining the Axion Window, Phys. Rev. Lett. 118 (2017) 031801 [arXiv:1610.07593] [INSPIRE].

[33] F. Arias-Aragon and L. Merlo, The Minimal Flavour Violating Axion, JHEP 10 (2017) 168 [Erratum ibid. 11 (2019) 152] [arXiv:1709.07039] [INSPIRE].

[34] G. Alonso-Álvarez, F. Ertas, J. Jaeckel, F. Kahlhoefer and L.J. Thormaehlen, Leading logs in QCD axion effective field theory, JHEP 07 (2021) 059 [arXiv:2101.03173] [INSPIRE].

[35] Y. Ema, K. Hamaguchi, T. Moroi and K. Nakayama, Flaxion: a minimal extension to solve puzzles in the standard model, JHEP 01 (2017) 096 [arXiv: 1612.05492] [INSPIRE].

[36] L. Calibbi, F. Goertz, D. Redigolo, R. Ziegler and J. Zupan, Minimal axion model from flavor, Phys. Rev. D 95 (2017) 095009 [arXiv: 1612.08040] [InSPIRE].

[37] L. Di Luzio, F. Mescia, E. Nardi, P. Panci and R. Ziegler, Astrophobic Axions, Phys. Rev. Lett. 120 (2018) 261803 [arXiv: 1712.04940] [INSPIRE].

[38] I.G. Irastorza and J. Redondo, New experimental approaches in the search for axion-like particles, Prog. Part. Nucl. Phys. 102 (2018) 89 [arXiv:1801.08127] [InSPIRE].

[39] J. Polchinski, String theory. Vol. 2: Superstring theory and beyond, Cambridge Monographs on Mathematical Physics, Cambridge University Press (2007) [DOI] [INSPIRE].

[40] J.P. Conlon, The QCD axion and moduli stabilisation, JHEP 05 (2006) 078 [hep-th/0602233] [INSPIRE].

[41] K. Choi and K.S. Jeong, String theoretic QCD axion with stabilized saxion and the pattern of supersymmetry breaking, JHEP 01 (2007) 103 [hep-th/0611279] [INSPIRE].

[42] K. Bobkov, V. Braun, P. Kumar and S. Raby, Stabilizing All Kähler Moduli in Type IIB Orientifolds, JHEP 12 (2010) 056 [arXiv:1003.1982] [INSPIRE].

[43] B.S. Acharya, K. Bobkov and P. Kumar, An M-theory Solution to the Strong CP Problem and Constraints on the Axiverse, JHEP 11 (2010) 105 [arXiv:1004.5138] [INSPIRE].

[44] M. Cicoli, M. Goodsell and A. Ringwald, The type IIB string axiverse and its low-energy phenomenology, JHEP 10 (2012) 146 [arXiv:1206.0819] [INSPIRE].

[45] K. Choi, K.S. Jeong and M.-S. Seo, String theoretic QCD axions in the light of PLANCK and BICEPQ, JHEP 07 (2014) 092 [arXiv: 1404.3880] [INSPIRE]. 
[46] M. Dine, N. Seiberg, X.G. Wen and E. Witten, Nonperturbative Effects on the String World Sheet, Nucl. Phys. B 278 (1986) 769 [INSPIRE].

[47] R. Blumenhagen, M. Cvetič, S. Kachru and T. Weigand, D-Brane Instantons in Type II Orientifolds, Ann. Rev. Nucl. Part. Sci. 59 (2009) 269 [arXiv:0902.3251] [inSPIRE].

[48] J.P. Conlon, D. Cremades and F. Quevedo, Kähler potentials of chiral matter fields for Calabi-Yau string compactifications, JHEP 01 (2007) 022 [hep-th/0609180] [INSPIRE].

[49] V. Balasubramanian, P. Berglund, J.P. Conlon and F. Quevedo, Systematics of moduli stabilisation in Calabi-Yau flux compactifications, JHEP 03 (2005) 007 [hep-th/0502058] [INSPIRE].

[50] E. Witten, Some Properties of O(32) Superstrings, Phys. Lett. B 149 (1984) 351 [InSPIRE].

[51] K. Choi and J.E. Kim, Compactification and Axions in $E_{8} \times E_{8}^{\prime}$ Superstring Models, Phys. Lett. B 165 (1985) 71 [inSPIRE].

[52] T. Banks and M. Dine, Couplings and scales in strongly coupled heterotic string theory, Nucl. Phys. B 479 (1996) 173 [hep-th/9605136] [INSPIRE].

[53] K. Choi, Axions and the strong CP problem in M-theory, Phys. Rev. D 56 (1997) 6588 [hep-th/9706171] [INSPIRE].

[54] P. Hořava and E. Witten, Eleven-dimensional supergravity on a manifold with boundary, Nucl. Phys. B 475 (1996) 94 [hep-th/9603142] [INSPIRE].

[55] A. Lukas, B.A. Ovrut and D. Waldram, On the four-dimensional effective action of strongly coupled heterotic string theory, Nucl. Phys. B 532 (1998) 43 [hep-th/9710208] [INSPIRE].

[56] J.P. Derendinger, L.E. Ibáñez and H.P. Nilles, On the Low-Energy Limit of Superstring Theories, Nucl. Phys. B 267 (1986) 365 [InSPIRE].

[57] L.E. Ibáñez and H.P. Nilles, Low-Energy Remnants of Superstring Anomaly Cancellation Terms, Phys. Lett. B 169 (1986) 354 [INSPIRE].

[58] E. Witten, Strong coupling expansion of Calabi-Yau compactification, Nucl. Phys. B 471 (1996) 135 [hep-th/9602070] [INSPIRE].

[59] N. Arkani-Hamed, G.F. Giudice, M.A. Luty and R. Rattazzi, Supersymmetry breaking loops from analytic continuation into superspace, Phys. Rev. D 58 (1998) 115005 [hep-ph/9803290] [INSPIRE]. 